\title{
Generalized Parton Distributions and Color Transparency Phenomena
}

\author{
S. Liuti ${ }^{1, *}$ and S. K. Taneja, 团 \\ ${ }^{1}$ University of Virginia, Charlottesville, Virginia 22904-4714, USA.
}

\begin{abstract}
We study the structure of generalized parton distributions in impact parameter space with the aim of determining the size and role of small transverse separations components in the quarks wave function. We analyze the relation between transverse momentum components and transverse separations. Wave functions with large transverse momentum components can simultaneously reproduce the behavior of the Dirac form factor at large momentum transfer, and of the deep inelastic structure functions at Bjorken $x \rightarrow 1$. The presence of large momentum components does not ensure, however, the dominance of small transverse distances at large $x$. We suggest that experiments measuring the attenuation of hadrons in the nuclear medium, or the onset of color transparency, can provide an alternative source of information on generalized parton distributions, by mapping out the behavior of the transverse components of the wave function.
\end{abstract}

PACS numbers: 13.60.Hb, 13.40.Gp, 24.85.+p

*s14y@virginia.edu

itskt6c@virginia.edu 


\section{INTRODUCTION}

A most intensively studied question in Quantum ChromoDynamics (QCD) is the spacetime structure of high energy exclusive reactions. In perturbative QCD (pQCD) approaches the hypothesis is made that elastic processes are dominated by the Fock space components of the hadronic wave function with the minimum number of quarks (anti-quarks). In the hard scattering picture these are assumed to be located within a small relative transverse distance $\lesssim 1 / \sqrt{Q^{2}}, Q^{2}$ being the (high) four-momentum transfer squared in the reaction [1]. After the hard scattering takes place, a normal hadron is recovered. The validation through experiments of this picture - the dominance of the short separation components - is however not straightforward, as witnessed for instance by the recent controversial interpretations of experiments sensitive to helicity selection rules [2, 3].

It has been long surmised [1, 4] that performing (quasi)-elastic reactions off nuclear targets might ease this impasse. Nuclei can in fact function as both "passive" or "active" probes for the small partonic separation components. Small distances can be filtered at finite $Q^{2}$, by impeding the passage of large separations because of the strong interactions occurring in a nucleus with $A>>1$. In this case nuclei play an active role [5]. One can also simply study the passage through the nuclear medium of the small separation component selected at asymptotically high $Q^{2}$, namely the phenomenon of Color Transparency (CT). $\mathrm{CT}$ is attained in principle when the ratio, $T_{A}\left(Q^{2}\right)=\sigma_{A} / A \sigma_{h}$, of the nuclear cross section to the one for scattering in free space, reaches the value of 1 at some given high value of $Q^{2}$. From a practical point of view, current searches for CT might appear to be in a stall as all experiments performed so far seem not to show either any systematics or any marked trend for the onset of this phenomenon [6]. However, at the same time, none of the experiments does contradict current predictions for CT. A large body of theoretical work exists, that generated an active field in the last decade (see [7, 8, 9] for extensive reviews). New observables have been proposed since the original measurements, such as the attenuation cross section [5], the systematics of oscillations in pion production reactions [10], or the sensitivity to the correlation length in exclusive vector meson production [11]. Based on these new hypotheses, more specific searches have been conducted, which in some cases seem to better identify the phenomenon [12, 13, 14, 15]. Critical observations about the key assumptions underlying the onset of CT have also recently emerged, a most disquieting one being the suggestion that the transverse size of the exclusive process might not even be small due to a "suppression of the Sudakov suppression" [16].

Whether or not a pQCD description of the hadrons holds at the $Q^{2}$ values presently available, it has now become imperative to investigate the basic question of the existence and observability of small size hadronic configurations. In this paper we use as a theoretical

tool Generalized Parton Distributions (GPDs) that, as pointed out recently [17], can give us direct access to the transverse spatial extension of the hadronic components. GPDs can, in fact, be shown to be diagonal in the impact parameter representation, and they can therefore be interpreted as - Fourier transformed in the transverse plane - joint probability distributions of finding a parton with longitudinal momentum fraction $x$, and transverse separation from the proton's center, $b[17$. The original result, obtained for $\xi=0, \xi$ being the longitudinal momentum transfer fraction, was shown to hold also in the $\xi \neq 0$ case in 18] (see also [19] for a laboratory frame based description).

The new insight provided by the impact parameter space interpretation allows one to make a connection between GPDs and the phenomena of CT and nuclear filtering. Within 
this new framework, one can in fact address directly the question of what transverse distances dominate the hard exclusive scattering cross section. So far, a quantitative knowledge of the size of the hadronic configurations involved in a reaction has been in fact very elusive, or at the best, loosely inferred from experiments.

This paper is organized as follows: in Section [1] we define GPDs and show their connection, at leading order in $Q^{2}$, to both the impact parameter dependent parton distributions, and to the intrinsic transverse momentum distributions. In Section [I] we evaluate the role of large transverse momentum components and, conversely, of small interparton separations. A few quantities are calculated, namely the GPDs at $\xi=0$, the Dirac form factor, and the the deep inelastic structure function. For each of these quantities the impact of different behaviors in the partons wave functions in both transverse momentum, and transverse distance, is evaluated. In Section IV we calculate the ratio $T_{A}\left(Q^{2}\right)$ for electron nucleus scattering experiments in terms of GPDs, and we suggest a method for extracting the transverse interparton distance distribution. Finally, we draw our conclusions in Section $\nabla$.

\section{SOME OLD AND NEW DEFINITIONS}

GPDs were initially introduced [20, 21] in order to better describe in a partonic language the concept of orbital angular momentum carried by the nucleon's constituents. They can be accessed experimentally in exclusive hard scattering experiments. The most straightforward way has been to date Deeply Virtual Compton Scattering (DVCS), that has become the prototype for illustrating different aspects of GPDs. There exist a few reviews to date on this subject 22, 23]. In this Section we summarize the properties of GPDs that are relevant for our discussion of exclusive scattering from nuclear targets, as e.g. probed in the reaction: $e A \rightarrow e^{\prime} p(A-1)$. In particular, we define:

- The Impact Parameter dependent Parton Distribution Function (IPPDF) and its relation to the observables, $F_{1}$, the Dirac form factor, $\nu W_{2}$, the DIS structure function, and $H$, the "non-spin flip" GPD.

- The rms impact parameter, interparton separation, and radius for the proton, in terms of both IPPDFs and GPDs.

- The intrinsic transverse momentum distribution, and its relation to both the IPPDFs, and to $F_{1}, \nu W_{2}$, and $H$.

The latter issue opens a new perspective on GPDs as "tools" to study transversity.

\section{A. Generalities}

We consider the leading order in $Q^{2}$ (twist-2), where one can define four universal GPDs, namely $H, E$ and $\widetilde{H}, \widetilde{E}$. The latter two are related to the quarks helicity distributions. In this paper we focus on the unpolarized case described by $H, E$. The relevant kinematical variables are: $P$ and $P^{\prime}$, the initial and final nucleon's momenta in the exclusive process, $\bar{P}=\left(P+P^{\prime}\right) / 2$, the average nucleon momentum, $k$ the active quark momentum, and $q$ the virtual photon momentum (Fig.1). While the (upper) hard scattering part of the diagram varies in principle for different reactions, the information necessary to describe GPDs is 
contained in the (lower) soft part (the different types of matrix elements related to GPDs are described e.g. in 22]). ${ }^{1}$ One can define four independent invariants using these variables: ${ }^{2} x=k^{+} / \bar{P}^{+}, \xi=-\Delta^{+} / 2 \bar{P}^{+}, Q^{2}=-q^{2}$, and $t \equiv \Delta^{2}$, where $\Delta=P-P^{\prime}$, is the four-momentum transfer in the process. Differently from inclusive Deep Inelastic Scattering (DIS), the four-momentum transfer, $\Delta$, does not generally coincide with the virtual photon four-momentum, $q$. By properly devising experimental measurements that reproduce this situation, namely $q \neq \Delta$, one has therefore the unique possibility of "zooming" in on partonic components, due to the high resolution provided by large $Q^{2}$ (in the multi-GeV region), at the same time scanning the hadronic target's spatial distribution, through the dependence on the variable $\Delta$. We denote by $H_{i}$ and $E_{i}$ the GPDs for a quark of type $i . H_{i}$ and $E_{i}$ satisfy the following relations:

$$
\begin{aligned}
& \int_{-1}^{1} d x H_{i}(x, \xi, t)=F_{1}^{i}(t), \\
& \int_{-1}^{1} d x E_{i}(x, \xi, t)=F_{2}^{i}(t),
\end{aligned}
$$

independently from $\xi$ (see [22, 23] $). F_{1(2)}^{i}$ are the Dirac and Pauli form factors for the quarks of type $i$ in the nucleon. They are readily obtained from the measured proton and neutron form factors by using isospin symmetry [22]. For $x \geq 0$,

$$
H_{i}(x, 0,0) \equiv q_{i}(x),
$$

i.e. one recovers the Parton Distribution Functions (PDFs), $q_{i}(x)$, in the in the forward limit. $E$ does not have an equivalent connection to parton distributions.

\section{B. Impact Parameter Space}

GPDs can be related [17] to the Impact Parameter space dependent PDFs (IPPDFs) (see e.g. Ref.[25]). An IPPDF is defined as the joint distribution: $d n_{i} / d x d \mathbf{b} \equiv q_{i}(x, \mathbf{b})-$ the number of partons of type $q$ with momentum fraction $x=k^{+} / P^{+}$, located at a transverse distance $\mathbf{b}$ ( $\mathbf{b}$ is the impact parameter) from the center of $P^{+}$of the system. ${ }^{3}$ The latter is defined as $\mathbf{R}=\left(\sum k_{n}^{+} \mathbf{b}_{n}\right) / P^{+}$, where $x_{n}^{\mu} \equiv\left(x^{0} ; \mathbf{x} \equiv \mathbf{b}, x^{3}\right)$ is the space-time variable for the $n-t h$ parton, and $\sum k_{n}^{+}=P^{+}$. The usage of Light Cone (LC) variables is motivated by the fact that by viewing $x^{+}$as the time variable, $P^{+}, p_{n}^{+}$as the total and partonic masses, respectively, and $\mathbf{R}$ as the center of mass of the system, $P^{+} \mathbf{R}$ is the generator of longitudinal boosts that leave the physics on the $x^{+}=0$ surface invariant. An intuitive connection with non-relativistic logics is naturally provided. The interpretation of observables, e.g. structure functions, in terms of parton density distributions relies on the validity of the Impulse Approximation (IA) whereby the hard scattering happens instantaneously in $x^{+}$. We work in this hypothesis by assuming that it is satisfied in the kinematical limit for the

\footnotetext{
${ }^{1}$ We do not take into consideration initial state nuclear medium modifications. These are part of a more general problem, and they will be addressed in a forthcoming paper 24.

${ }^{2}$ We use the notation: $a^{\mu} \equiv\left(a^{0} ; \mathbf{a}_{\perp}, a^{3}\right)$, and $a^{ \pm}=\left(a^{0} \pm a^{3}\right)$

3 All transverse variables are denoted here by bold-face characters.
} 
coherence length of the virtual photon given by: $x^{+} \ll L \approx 1 / 2 M_{N} x_{B j}$. This amounts to considering only large values of $x_{B j}$. At low $x_{B j}$, one has to consider both IA-type scattering and of processes including photon splitting into quark-antiquark pairs before the nucleon where it has been recently shown that FSI might hamper the interpretation of the deep inelastic measurements in terms of probability distributions [26]. This is part of a wider set of problems that transcend the goals of this paper. In particular, it will be important to study in the future whether parton interactions (or off-shellness) affect the transverse distributions that we address in this paper, as this might complicate the procedure of extracting holographic images of the proton as proposed in [19, 27].

By definition [25], The DIS structure function, $\nu W_{2}(x)$, and the form factor, $F_{1}\left(Q^{2}\right)$, can be written in terms of the IPPDFs respectively, as:

$$
\begin{aligned}
\nu W_{2}(x) & =\sum_{i} e_{i}^{2} x \int d^{2} \mathbf{b} q_{i}(x, \mathbf{b}) \\
F_{1}(\Delta) & =\sum_{i} e_{i} \int d^{2} \mathbf{b} e^{i \boldsymbol{\Delta} \cdot \mathbf{b}} \int_{0}^{1} d x q_{i}(x, \mathbf{b}),
\end{aligned}
$$

where $e_{i}$ is the quark's charge, and we have disregarded the $Q^{2}$ dependence of $\nu W_{2}$. Notice that $\Delta$ is the conjugate variable of $\mathbf{b}$.

\section{1. $\mathbf{b}$ distribution}

In [17], the observation was made that for a purely transverse four momentum transfer, namely for $\Delta \equiv\left(\Delta_{0}=0 ; \boldsymbol{\Delta}, \Delta_{3}=0\right)$ and $\xi=0, H_{i}\left(x, 0,-\boldsymbol{\Delta}^{2}\right)$, and $q_{i}(x, \mathbf{b})$ can be related as follows:

$$
\begin{aligned}
q_{i}(x, \mathbf{b}) & =\int \frac{d^{2} \boldsymbol{\Delta}}{(2 \pi)^{2}} e^{-i \mathbf{b} \cdot \boldsymbol{\Delta}} H_{i}\left(x, 0,-\boldsymbol{\Delta}^{2}\right) \\
H_{i}\left(x, 0,-\boldsymbol{\Delta}^{2}\right) & =\int d^{2} \mathbf{b} e^{i \mathbf{b} \cdot \boldsymbol{\Delta}} q_{i}(x, \mathbf{b})
\end{aligned}
$$

Since $q_{i}(x, \mathbf{b})$ satisfies positivity constraints and it can be interpreted as a probability distribution, $H_{i}\left(x, 0,-\boldsymbol{\Delta}^{2}\right)$ is also interpreted as a probability distribution, namely the Fourier transformed joint probability distribution of finding a parton $i$ in the proton with longitudinal momentum fraction $x$, at the transverse position $\mathbf{b}$, with respect to the center of momentum of the nucleon. Since in what follows we will consider only the case $\xi=0$, we define: $H_{i}\left(x, 0,-\boldsymbol{\Delta}^{2}\right)=H_{i}(x, \boldsymbol{\Delta})$.

The root-mean-square (rms) distance of the configuration with momentum fraction $x$, from the center of the proton is defined as the square root of:

$$
\left\langle\mathbf{b}^{2}(x)\right\rangle=\mathcal{N} \int d^{2} \mathbf{b} q(x, \mathbf{b}) \mathbf{b}^{2}
$$

$\mathcal{N}$ being a normalization factor, i.e. $\mathcal{N}^{-1}=\int d^{2} \mathbf{b} q(x, \mathbf{b})$. Similar relations to the ones in Eqs. 4a 4b), were obtained for $\xi \neq 0$ in [18]. An alternative approach in terms of Wigner distributions, formulated in a covariant model that allows one in principle to perform calculations in the laboratory frame was given in [19]. The role of the variable $\xi$ in the context 
of a covariant approach, is an important subject per se, especially in reference to Initial State Interactions (ISI) in nuclei, since it impacts the particles' off-shellness. We address this subject in detail in [24].

\section{2. $\mathbf{y}$ distribution and hadronic radius}

One can also define the Fourier transform with respect to the variable $\mathbf{y}=\mathbf{b} /(1-x)$, the transverse position of parton $i$ with respect to the center of momentum of the system of spectator partons [25] :

$$
\mathcal{H}_{i}(x, \widetilde{\mathbf{k}})=\int d^{2} \mathbf{b} e^{i \widetilde{\mathbf{k}} \cdot \mathbf{y}} q(x, \mathbf{b})
$$

$\mathcal{H}$ can be obtained from $H$ by rescaling the functions arguments as follows:

$$
H_{i}(x, \boldsymbol{\Delta}) \equiv \mathcal{H}_{i}(x, \boldsymbol{\Delta}(1-x))
$$

Since $\mathbf{y}$ measures the interparton separation, it is useful to define also the inverse Fourier transform:

$$
\widetilde{\mathcal{H}}_{i}(x, \mathbf{y})=\int d \mathbf{k}^{\prime} e^{-\tau \tilde{\mathbf{k}} \cdot \mathbf{y}} \mathcal{H}_{i}(x, \widetilde{\mathbf{k}}) \equiv q(x, \mathbf{y}(1-x)) .
$$

The variables $\widetilde{\mathbf{k}} \equiv \boldsymbol{\Delta}(1-x)$ and $\mathbf{y}$ are Fourier conjugates, as well as $\boldsymbol{\Delta}$ and $\mathbf{b} \equiv \mathbf{y}(1-x)$. $\widetilde{\mathcal{H}}(x, \mathbf{y})$ is the probability of finding a parton with momentum fraction $x$, at a transverse distance $\mathbf{y}$ from the center of momentum of the spectator partons. The rms transverse distance between partonic configurations with momentum fractions $x$ is obtained as the square root of:

$$
\left\langle\mathbf{y}^{2}(x)\right\rangle=\mathcal{N} \int d^{2} \mathbf{b} q(x, \mathbf{b}(\mathbf{y})) \mathbf{y}^{2},
$$

i.e. from the same observable $H$ used to obtain $\left\langle\mathbf{b}^{2}\right\rangle . \mathcal{N}$ is defined analogously to Eq.(15).

The radius of the system of partons, which is the useful quantity for our CT study, is:

$$
\left\langle\mathbf{r}^{2}(x)\right\rangle^{1 / 2}=M A X\left\{\left\langle\mathbf{b}^{2}(x)\right\rangle^{1 / 2},\left\langle\mathbf{b}^{2}(x)\right\rangle^{1 / 2} \frac{x}{1-x}\right\}
$$

This definition coincides with the one given in [17] for $x \rightarrow 1$. In Fig 2 we sketch a cartoon of the different transverse distances discussed so far. The figure shows three different scenarios for the behavior of such distances with increasing $x$, corresponding to the models discussed in the following Sections.

\section{Intrinsic transverse momentum distribution and spectral function}

The function $\mathcal{H}_{i}(x, \widetilde{\mathbf{k}})$ defined in the previous subsection, although being related through a Fourier transform to the interparton separation, $\mathbf{y}$, is conceptually different from the "intrinsic transverse momentum" distribution.

Although we started from writing the structure function and the form factor in terms of IPPDFs (Eqs. (3a) and (3b), respectively), their definition is usually given in terms of a LC 
wave function, $\phi(x, \mathbf{k})$ as [28]:

$$
\begin{aligned}
\nu W_{2}(x) & =\sum_{i} e_{i}^{2} x \int d^{2} \mathbf{k} f(x, \mathbf{k}) \\
F(\boldsymbol{\Delta}) & =\sum_{i} e_{i} \int d^{2} \mathbf{k} \int_{0}^{1} d x f(x, \mathbf{k}, \mathbf{k}+(1-x) \boldsymbol{\Delta}),
\end{aligned}
$$

where:

$$
f\left(x, \mathbf{k}, \mathbf{k}^{\prime}\right)=\phi^{*}(x, \mathbf{k}) \phi\left(x, \mathbf{k}^{\prime}\right),
$$

with $\mathbf{k}^{\prime} \equiv \mathbf{k}+(1-x) \boldsymbol{\Delta}$, is a non-diagonal intrinsic momentum distribution. The diagonal term can be written as:

$$
f(x, \mathbf{k})=|\phi(x, \mathbf{k})|^{2} .
$$

By comparing Eqs.(11a, 11b), with Eqs.(3a, 3b), we find that the relation between the transverse momentum and the transverse separation of quarks inside a hadron is obtained through a non-diagonal distribution in transverse coordinate space, $q\left(x, \mathbf{b}, \mathbf{b}^{\prime}\right)$ :

$$
f(x, \mathbf{k})=\int d^{2} \mathbf{b} \int d^{2} \mathbf{b}^{\prime} e^{i \mathbf{k} \cdot\left(\mathbf{b}-\mathbf{b}^{\prime}\right)} q\left(x, \mathbf{b}, \mathbf{b}^{\prime}\right)
$$

where we define:

$$
\begin{aligned}
q\left(x, \mathbf{b}, \mathbf{b}^{\prime}\right) & =\Psi^{*}\left(x, \mathbf{b}^{\prime}\right) \Psi(x, \mathbf{b}) \\
q(x, \mathbf{b}, \mathbf{b}) & =|\Psi(x, \mathbf{b})|^{2} \equiv q(x, \mathbf{b}),
\end{aligned}
$$

$\Psi(x, \mathbf{b})$ being a wave function in the mixed $x$ and $\mathbf{b}$ representation, related to the $\mathrm{LC}$ wave function by a transverse Fourier transform. This result can be also implemented in studies of processes directly sensitive to $\mathbf{k}$. It is useful for this purpose to consider the average transverse momentum squared, defined as:

$$
\left\langle\mathbf{k}^{2}(x)\right\rangle=\mathcal{N} \int d^{2} \mathbf{k} f(x, \mathbf{k}) \mathbf{k}^{2}
$$

with $\mathcal{N}^{-1}=\int d^{2} \mathbf{k} f(x, \mathbf{k})$. In Section III we present an initial exploratory study of the relative roles of the intrinsic transverse momentum and the transverse separation using different types of wave functions.

Finally, it should be mentioned that Eq.(11a) corresponds to a projection obtained from the partons covariant spectral function, $S(k)[29$, 30, 31]:

$$
\begin{aligned}
\nu W_{2}(x) & =\sum_{i} e_{i}^{2} x \int d^{4} k S(k) \delta\left(x-\frac{k^{+}}{P^{+}}\right) \\
& =\sum_{i} e_{i}^{2} x \int d^{2} \mathbf{k} \int d P_{X}^{2} S(k),
\end{aligned}
$$

where the integration over $k^{-}$in the last line is replaced by an integration over the invariant mass of the spectator system, $P_{X}^{2}=(P-k)^{2}\left(k^{-}\right.$and $P_{X}^{2}$ are related by). The main reason for introducing the spectral function along with the intrinsic momentum distributions is 
that it encodes information about the energy and momentum of the spectator system. The spectral function can be defined in fact as the joint probability distribution of finding a parton with LC components $k^{+}$and $\mathbf{k}$, leaving the spectator system with a mass $M_{X}^{2} \equiv P_{X}^{2}$. The usage of the spectral function is most important in problems where the particles' offshellness/transversity plays a role, a typical example being deep inelastic scattering from nuclei [24, 29, 30, 31]. In the following Sections we show a model of GPDs that implements a function $S(k)$ within relativistic IA.

\section{MODELS FOR IMPACT PARAMETER DEPENDENT PARTON DISTRIBU- TION FUNCTIONS}

We evaluate the functions defined in the previous Section at leading order in $Q^{2}$, using a two component, or spectator, model (Fig.1b). The main assumptions of the model are: $i$ ) the validity of the hand-bag diagram; ii) a description of dynamics in terms of a nucleon-quarkspectator system vertex. The minimal Fock component is described by a quark-diquark vertex function. We describe the active quark as off-shell and the diquark as on-shell, with $\operatorname{mass} M_{X}=\sqrt{P_{X}^{2}}$.

The spectator model is both widely used and well tested in hadronic physics. In particular, in [29] it was used to describe DIS in nuclei; in [30] parametrizations for both the polarized and unpolarized proton and neutron structure functions were given; more recently it was applied to DVCS [32, 33]. Our usage of the model other than for practical reasons, is motivated by the clear handling of the interplay between the particles' off-shellness and their energy and transverse momentum. This turns out to be an important property when describing the deep inelastic structure of nuclei [24]. By considering the components in Fig.1b, one has

$$
\begin{aligned}
k^{2} & =k^{+} k^{-}-\mathbf{k}^{2}= \\
& =\left(x P^{+}\right)\left[\frac{M^{2}}{P^{+}}-\frac{M_{X}^{2}+\mathbf{k}^{2}}{(1-x) P^{+}}\right]-\mathbf{k}^{2} \\
k^{\prime 2} & =k^{\prime+} k^{\prime-}-(\mathbf{k}+\boldsymbol{\Delta})^{2}= \\
& =\left(x P^{+}\right)\left[\frac{M^{2}+\Delta^{2}}{P^{+}}-\frac{M_{X}^{2}+\mathbf{k}^{2}}{(1-x) P^{+}}\right]-(\mathbf{k}+\boldsymbol{\Delta})^{2}
\end{aligned}
$$

where $\boldsymbol{\Delta}^{2} \equiv-t ; M$ and $M_{X}$ are the proton and the diquark masses, respectively. Hence, for the denominators in Fig.1b:

$$
D(x, \mathbf{k})=\mathcal{M}_{X}^{2} x-\frac{\mathbf{k}^{2}}{1-x},
$$

where we defined $\mathcal{M}_{X}^{2}=M^{2} x-M_{X}^{2} /(1-x)-m^{2} / x, m$ being the struck quark mass. We consider the remnant diquark system to be a scalar. The form of the vertex function depends on the assumptions made in treating the quark's spin. Since we are ultimately interested in determining the radius of the proton as extracted by using the spin averaged quantity $H$, we assume that the vertex function is a scalar in Dirac space described by a function $g\left(k^{2}\right)$. The two-component wave function becomes:

$$
\phi(x, \mathbf{k})=\frac{g\left(k^{2}\right)}{D(x, \mathbf{k})} .
$$


Notice that, because of the relation between the struck parton's virtuality and transverse momentum (Eq. (18b) ), one can write $\phi$ as a function of the invariants: $k^{2}, x, M_{X}^{2}$. One can therefore identify the relativistic spectral function in Eq.(17) with:

$$
S(k)=\left|\phi\left(k^{2}, M_{X}^{2}, x\right)\right|^{2} .
$$

\section{A. GPD, Structure Function, and Form Factor}

The GPD, the structure function, and the form factor can be evaluated in terms of the function $\phi$ in Eq.(20), according to the definitions in Section 1 113 One has:

$$
\begin{aligned}
H(x, \boldsymbol{\Delta}) & =\int d^{2} \mathbf{k} \phi^{*}(x, \mathbf{k}) \phi(x, \mathbf{k}+(1-x) \boldsymbol{\Delta}) \\
\nu W_{2}(x) & =\int d^{2} \mathbf{k}|\phi(x, \mathbf{k})|^{2} \\
F_{1}\left(\boldsymbol{\Delta}^{2}\right) & =\int d^{2} \mathbf{k} \int_{0}^{1} d x \phi^{*}(x, \mathbf{k}) \phi(x, \mathbf{k}+(1-x) \boldsymbol{\Delta}),
\end{aligned}
$$

Notice that we incorporated in the function $\phi$, a factor $\sqrt{x /(1-x)}$, that accounts for both the initial flux, and the phase space for the process in Fig.1. Moreover, we drop the index $i$ in $H$, since we fit our functions directly to the proton valence structure function and form factor, without distinguishing between $u$ and $d$ quarks contributions. For the vertex function we used the form:

$$
g\left(k^{2}\right)=g \frac{\Lambda^{2}-m^{2}}{k^{2}-\Lambda^{2}},
$$

where $g$ is fixed by the normalization, and $\Lambda$ is a cut-off parameter. The values of $\Lambda, m$, and $M_{X}$ are determined by fitting both the large four-momentum behavior of the form factor, and the shape of the DIS structure function at large $Q^{2}$. The latter is determined in our model calculation at very low $Q^{2}\left(Q^{2}<1 \mathrm{GeV}^{2}\right)$, where the structure function is given only by its valence component, the sea quarks and gluons being generated through perturbative evolution (see e.g. 34]). The values of the parameters are: $m=0.3 \mathrm{GeV}$, $M_{X}=1.100 \mathrm{GeV}, \Lambda=0.73 \mathrm{GeV}$. In Figures 3, 4, 5, and 6 we present our results obtained using Eqs. (20122a 22b 22c 23).

Fig. 3 shows the quantity: $|\Phi(\mathbf{k})|^{2}$, where

$$
\Phi(\mathbf{k})=\int_{0}^{1} d x \phi(x, \mathbf{k}) .
$$

Also shown for comparison are results obtained using the parametrizations from Refs. 35, 36, 37]. The wavefunction in Eq. (20), has a similar behavior to the parametrization used in 36], in that it displays high momentum components with a $\propto 1 / \mathbf{k}^{4}$ behavior, although in a slightly larger amount. In order to quantify the "hardness" of the distributions, in Table [1 we show the average transverse momentum values obtained in each model. In the Table we also display the contributions to $\left\langle\mathbf{k}^{2}\right\rangle$, from the large momentum components calculated by setting the lower limit of integration in $|\mathbf{k}|$ equal to $1 \mathrm{GeV}$ and to $2 \mathrm{GeV}$, respectively. These values reflect the behavior of the curves in Fig [3, in that the distribution in Ref. 21] is truly of the "soft" type; the ones in Ref. [36] and in this paper are characterized by a long 
TABLE I: Contribution of large transverse momentum components from different GPD models: values of $\left\langle k^{2}\right\rangle$ (first column); percentage contribution to $\left\langle k^{2}\right\rangle$ from $k>1 \mathrm{GeV}$ components (second column), and from $k>2 \mathrm{GeV}$ components (third column).

\begin{tabular}{|c|c|c|c|}
\hline & $\left(\left\langle k^{2}\right\rangle^{1 / 2} \mathrm{MeV}\right)$ & $\%$ value for $k_{\min }=1(\mathrm{GeV})$ & $\%$ value for $k_{\min }=2(\mathrm{GeV})$ \\
\hline \hline This paper & 360 & 14 & 1.5 \\
Radyushkin '98 [35] & 290 & 0.1 & 0 \\
Stoler '02 [36] & 286 & 2.0 & 1.0 \\
Burkardt '04 [37] & 415 & 15 & $6 \times 10^{-2}$ \\
\hline
\end{tabular}

high momentum "tail"; finally, we show results obtained with parametrizations of the type proposed in Ref. [37], where by accounting for gluonic interactions through the introduction of an effective gluonic mass in the initial two component model of [35], a further suppression in the large $x$ behavior is found, of the type $\approx \exp \left[(1-x)^{n} \Delta^{2}\right]$, with $n \geq 2$. We find that for $n=2,3$ the distributions obtained following the idea of Ref. [37], are neither entirely "soft" nor "hard", but they display larger "intermediate" momentum values, hence they give rise to a large $\langle\mathbf{k}\rangle^{2}$.

The function $H(x, \boldsymbol{\Delta})$ is shown in Fig.4, plotted vs. $\boldsymbol{\Delta}$ for different values of $x$. A small $(x=0.07)$, an intermediate $(x=0.36)$ and a large $(x=0.88)$ value of $x$ are indicated in the figure, from which one can also deduce that the value of $H(x, 0)$ traces the expected behavior for the valence structure function (namely the curve at $x=0.07$ has a much lower value for $\Delta \rightarrow 0$ than the curve at $x=0.36)$. Values of $x \gtrsim 0.9$ decrease one more order of magnitude and they are not shown in the figure.

In Fig. 5 we show the form factor $F_{1}\left(\Delta^{2}\right)$. This is compared to calculations using the models of Ref. 21], Ref.[36], and to the dipole model: $F_{1}\left(\Delta^{2}\right)=\mathcal{F} 1 /\left(1+\Delta^{2} / 0.71\right)^{2}$, with $\mathcal{F}=\left(\Delta^{2} / 4 M^{2} \mu_{P}+1\right) /\left(1+\Delta^{2} / 4 M^{2}\right)$. We find, in agreement also with Ref. [36], that the large momentum components of the wave function are responsible for the large $\Delta^{2}$ behavior of the form factor. Such components are effectively comparable to the hard contribution from pQCD calculations 32]. Although they constitute a small fraction of the average transverse momentum as shown in Table I, the contribution of large $\mathbf{k}$ is much larger in the nucleon form factor. This is shown in Fig.6, where we plotted the ratio: $F_{1}\left(\boldsymbol{\Delta}, \mathbf{k}_{\max }\right) / F_{1}\left(\boldsymbol{\Delta}, \mathbf{k}_{\max }=\infty\right)$. Only $\approx 70 \%$ of the form factor is reproduced by stopping the integration at $k_{\max }=0.7 \mathrm{GeV}$, by using a wave function with hard components, whereas the ratio shown in the figure is already saturated in models based on a Gaussian behavior.

Similar conclusions can be drawn by investigating the large $x$ behavior of the deep inelastic structure function, Eq. (22b).

\section{B. Impact Parameter Space Parton Distributions}

Next, we study how the behavior of the wave function with transverse momentum, $\mathbf{k}$, reflects on the transverse space distribution, $q(x, \mathbf{b})$. This is obtained as a Fourier transform 
of the function $H(x, \boldsymbol{\Delta})$ in the transverse plane: ${ }^{4}$

$$
q(x, b)=\frac{1}{2 \pi} \int_{0}^{\infty} d \Delta \Delta J_{0}(b \Delta) H(x, \Delta)
$$

where $\Delta=|\boldsymbol{\Delta}|$ and $b=|\mathbf{b}|$, and $J_{0}$ is a zero-th order Bessel function of the first kind. In order to evaluate $q(x, b)$ with adequate accuracy, we parametrized $H(x, \Delta)$ as:

$$
H(x, \Delta)=\frac{p_{1}(x)}{\left(p_{2}(x)+p_{3}(x) \Delta^{2}+p_{4}(x) \Delta\right)^{2}}
$$

The coefficients $p_{i}, i=1,4$ are given in the Appendix. The Hankel transform of $H$ has the following analytic form:

$$
q(x, b)=\frac{1}{2} b \frac{p_{1}(x) p_{2}(x)}{\sqrt{p_{3}(x) / p_{2}(x)}} K_{1}\left(\sqrt{p_{2}(x) / p_{3}(x)} b\right),
$$

where $K_{1}$ is a modified Bessel function of the second kind, and we set $p_{4}=0$ (a more complicated, though similar, analytical form is obtained for $p_{4} \neq 0$, which we do not display for ease of presentation). $q(x, b)$ is shown in Fig.7, plotted as a function of $b$ for varying $x$.

Using Eq.(27) one can calculate the radius, the interquark separation, and the rms distance from the center of the hadron, Eqs.(5910). These quantities are shown in Fig.8. In Fig.8(a) results for $\left\langle b^{2}\right\rangle^{1 / 2}$ from several models for the GPDs are compared. The difference in slope as $x \rightarrow 1$ is clearly visible. In Fig.8(b) we show our results for $\left\langle b^{2}\right\rangle^{1 / 2},\left\langle y^{2}\right\rangle^{1 / 2},\left\langle r^{2}\right\rangle^{1 / 2}$. One can see that the both rms radius, and the interparton separation do not vanish in the $x \rightarrow 1$ limit; rather, they tend to a finite (small) value of $\approx 2.5 \mathrm{GeV}^{-1}(\approx 0.5 \mathrm{fm})$. In Fig.9 we plot the average transverse momentum squared of a quark in the proton, Eq. (16). There is a marked difference in the behavior of this quantity for the "soft" and "hard" type wave functions. The wave function proposed in 37] gives manifestly divergent results for large $x$, although, as one can see from Table I, the integrated value of $\left\langle\mathbf{k}^{2}\right\rangle$ is finite. Our results are consistent with previous findings using the diquark/spectator model [30].

What components of the wave function are responsible for this behavior? In Fig.10 we give a partial answer to this question by plotting the contribution to both $b$ (upper panel) and $r$ (lower panel) of the short distance components. This is obtained by stopping the integration over $b$ in the definition of the rms quantities at decreasing values of the upper limit: 2, 1, and $0.5 \mathrm{GeV}^{-1}$, respectively. It is clear that the short distance part of the wave function (or the large $k$ components) largely determine the size of the radius at large $x$. This is particularly evident from the lower panel of Fig.10, where results are presented using the ratio: $\left\langle r^{2}\left(b_{\max }\right)\right\rangle /\left\langle r^{2}(\infty)\right\rangle$. While models including a hard component saturate this ratio for $b<0.5 \mathrm{GeV}^{-1}$ already at $x=0.8$, models using a "soft" wave function of the type of Ref. [35] do not show a tendency to saturation until very large $x$. These results open up a somewhat puzzling situation: In order to describe the form factor at large $Q^{2}$, one needs high $k$ components in the wave function. This is both a prediction of pQCD based calculations [2, 3], and it can be also shown phenomenologically (Fig.5) (see also Ref. [36]). High $k$ components are also responsible for a decrease in the hadronic transverse size as $x$ increases (Figs.8, 9 and 10). However, one can envisage a model such as the one utilized in

\footnotetext{
${ }^{4}$ For a circularly symmetric function of $\Delta$ this is a Hankel transform
} 
this paper that has the amount of high momentum components necessary to reproduce the Dirac form factor, and that at the same time, predicts a small but not vanishing transverse size for hadronic configurations at large $x$.

In concluding this Section, we reiterate that the usage of GPDs for determining the spatial structure of hadrons through $q(x, b)$, has been so far largely theoretical, since these quantities are only indirectly deduced from a variety of delicate measurements (38, 39]). In the following we suggest that an additional source of information could be given by studies of Color Transparency and related phenomena such as Nuclear Filtering. These are in fact directly sensitive to the size of hadronic components.

\section{EXCLUSIVE SCATTERING IN THE NUCLEAR MEDIUM}

In the previous Sections we discussed the relative importance of the soft and hard components of the light cone wave functions up to four momentum transfer $Q^{2}=25-30 \mathrm{GeV}^{2}$. Plausible scenarios confirm the idea that $\approx 30 \%$ of the form factor is given by the hard component of the wave function at four momentum transfer $Q^{2}=25-30 \mathrm{GeV}^{2}$ (for consistency with the general notation we switch from $\Delta^{2}$ to $Q^{2}$ in this Section). This result is indeed correlated with the dominance of small transverse sizes of hadronic configurations through a Fourier transform, however not straightforwardly as demonstrated by the different behaviors of the GPDs with $x$ and $\Delta$. As shown in Fig.11 where the average value of $x$, given by:

$$
x_{\text {ave }}(\Delta)=\frac{\int_{0}^{1} d x x H(x, \Delta)}{\int_{0}^{1} d x H(x, \Delta)},
$$

is plotted vs. $\Delta \equiv \sqrt{Q^{2}}$, the model in Ref. [35] is governed at large $Q^{2}$ by small $b$ as well as by large $x$ components (although its prediction for the form factor is substantially lower than the data, as shown in Fig [5] and the radius predicted for hadronic configurations diverges at large $x$ ). The approach based on the diquark model yields a form factor that is dominated by small $b$ components but not exclusively by $x \rightarrow 1$ configurations. This behavior which is further clarified by the comparison of $H(x, \Delta)$ for the two cases in Fig.12, is at the origin of the rather flat dependence with $x$ of the transverse radius shown in Fig.8. Also, it appears that the model of Ref. [37], approximately including the effect of a gluonic interaction, fixes the problem of an unphysically large radius while keeping a large $x$ behavior as the one in Ref. [35], at the expense of introducing a large $k$ in the wavefunction (Fig 91).

In summary, while it can be challenging to unambiguously disentangle the amount and nature of hard components responsible for the large $Q^{2}$ behavior of the hadronic form factors, one might gain a better insight by requiring models to simultaneously describe the hadrons transverse spatial distribution, and in particular the possible onset of small transverse configurations. The diquark model presented in this paper seems to provide a better description than other models similarly based on the handbag diagram.

If configurations with small radii indeed exist, they can be isolated in principle by performing CT and/or nuclear filtering type experiments. CT has been so far investigated in a variety of reactions including proton-hadron, $\gamma$-hadron, and electron-proton scattering 6, 12, 13, 14]. Here we consider exclusive electron-proton scattering, where oscillations due to the interference of perturbative and non-perturbative contributions possibly present in the proton-proton and $\gamma$-proton exclusive cross sections, do not occur. Our result is however quite general and can be easily extended to other type of reactions. 
Using the new perspective of GPDs in impact parameter space, we can now take a radical turn and devise a "minimal" approach that reduces the (nuclear) model dependence of the problem (an approach complementary to ours was recently suggested in [46], where the connection between CT and GPDs was explored in relation to diffractive dissociation of pions). As widely underlined in the literature, the prerequisites for the onset of CT, assuming that the scattering takes place off a small size configuration, are that: i) the interaction of the hadron in the nuclear medium is reduced due to the decreased gluon radiation from a small transverse size color dipole [40, 41]; ii) the small size configuration, not being stationary, will in principle evolve with time into a larger configuration. It however remains sufficiently small during the time it crosses the nucleus. Point ii) has probably been the most controversial one, although recent criticism to i) has also emerged [16]. Another point, not extensively addressed in the literature (see however [8]), is that the hadron suffers Initial State Interactions (ISI), or that in other words, the form factor for an off-shell nucleon is in principle different from the on-shell one.

We can now break down the problem in several steps: We first assume that the scattering happens with an "unmodified" proton in the nuclear medium. We introduce a nuclear filter for the large transverse size components, by multiplying the IPPDF, $q(x, b)$, by a square function:

$$
\Pi(b)= \begin{cases}1 & b<b_{\max }(A) \\ 0 & b \geq b_{\max }(A)\end{cases}
$$

where $b_{\max }(A)$ defines the size of the filter. This affects the GPD as:

$$
H_{A}\left(Q^{2}\right)=\int_{0}^{b_{\max }(A)} d b b q(x, b) J_{0}(b \Delta)
$$

with $\Delta=\sqrt{Q^{2}}$. The transparency ratio for $\left(e, e^{\prime} p\right)$ type reactions becomes

$$
T_{A}\left(Q^{2}\right)=\frac{\left[\int_{0}^{1} d x H_{A}(x, \Delta)\right]^{2}}{\left[\int_{0}^{1} d x H(x, \Delta)\right]^{2}},
$$

where $H$ defined as in Eq. (4b) represents scattering in free space, i.e. it is calculated with $b_{\max }=\infty$. Based on this result, one can fit the available data, using different distributions $q(x, b)$, and varying the parameter $b_{\max }$. In Fig 13 we show a few possible scenarios based on a simplified analytical model for $q(x, b)$. We take $q(x, b)=A(x) \exp (-\alpha(x) b)$, where $\alpha(x)$ is taken with two different dependencies: (a) $\alpha \propto(1-x) / x$, as in Ref. 35]; (b) $\alpha \propto(1-x)^{2}$, as in Ref.[37]. The effect of the filter is to produce both damping and oscillations in $H_{A}$. In Fig 13 we show for illustration, the ratio $R=H_{A}(x, \Delta) / H(x, \Delta)$ plotted vs. $\Delta$ for two different values of $x$, in case $(\mathbf{a})$ and $(\mathbf{b})$, and for different values of the filter size. Our analytical calculation displays explicitly the damping and oscillations as:

$$
R=1-\exp \left(-\alpha b_{\max }\right)\left(\alpha b_{\max } J_{0}\left(\Delta b_{\max }\right)+\cos \left(\Delta b_{\max }\right)\right) .
$$

Although this result does not correspond to a completely realistic situation, it allows us to understand for varying $x$, the different effects due to variations in the size of $\alpha$, which in turn is a feature of GPDs from different models. Oscillations are clearly more pronounced at low $x$. When $b_{\max }$ is large, of the order of $5 \mathrm{GeV}^{-1}(\approx 1 \mathrm{fm})$, transparency is attained. 
When $b_{\max }$ is small $\left(1 \mathrm{GeV}^{-1}, \approx 0.2 \mathrm{fm}\right)$, the medium is no longer transparent to the type of distributions considered, although distribution (a) giving a larger radius, is more suppressed than (b) at low $\Delta$.

The results of the first step of the analysis would give joint information on possible sizes of the filter, defined as $y_{\max } \equiv b_{\max } /(1-x)$, and on the behavior of the IPPDFs, i.e. on the radius of hadronic configurations, $\left\langle\mathbf{r}^{2}\right\rangle^{1 / 2}$.

This procedure, systematically applied to a sufficiently large body of data including both existing and planned measurements [6, 13, 14, 42], gives a much more direct test of the transverse sizes involved. Once this basic information is known from measurements - and not inferred from theoretical scenarios - one would be able to introduce more sophisticated modeling of e.g. rescattering and ISI. A somewhat similar point of view was taken in a pre-GPD context in Ref. 43] and applied to the data in [44, 45], where fits were performed based on a parameter, $p=n_{A} R_{A} \sigma_{e f f}$, with $n_{A}$ the nuclear density, $R_{A}$ the nuclear radius, and $\sigma_{\text {eff }}$ the effective hadron-hadron interaction cross section.

\section{CONCLUSIONS AND OUTLOOK}

We presented a study using the new theoretical insight provided by GPDs, of the interplay between the transverse variables in both momentum and coordinate space, $\mathbf{k}$ and b respectively, and of the longitudinal momentum fraction, $x$, in the proton form factor, $F_{1}$. Our study was aimed at establishing what type of components - hard vs. soft, small transverse distance vs. large - dominate the form factor based on different hypotheses for the GPDs used to describe $F_{1}$.

We examined three types of distributions (Figs 3 and 4 ):

i) Parametrizations based on Gaussian wave functions with argument $\propto(1-x) \Delta^{2}[\underline{35}$;

ii) Parametrizations based on Gaussian wave functions with argument $\propto(1-x)^{n} \Delta^{2}$, with $n \geq 2,[37$;

iii) GPDs obtained using a diquark/spectator model consistent with a $1 / k^{4}$ asymptotic dependence of the wavefunction.

We confirm the result initially obtained in Ref. 36] (Fig. [5) that $F_{1}$ at $\Delta>2 \mathrm{GeV}$ can be reproduced only by allowing for a sufficiently large amount of large $\mathbf{k}$ (hard) components. Cases ii), and iii) satisfy this condition. Furthermore, we find that, for values of $\Delta^{2} \approx 25-30$ $\mathrm{GeV}^{2}$, the behavior predicted for the form factor (Figs 6, 9, 11) is governed by:

i) Large $x\left(x_{\text {ave }}(\Delta=5 \mathrm{GeV}) \approx 0.75\right), k \lesssim 0.7 \mathrm{GeV}$ in $[35]$;

ii) $x \rightarrow 0$, large $k$ in 37 ;

iii) $x \approx 0.25, k \lesssim 2 \mathrm{GeV}$ in our diquark/spectator model.

The rms value of the proton radius, given by $\left\langle\mathbf{r}^{2}\right\rangle^{1 / 2}$ in Eq.(10), was calculated by a two-dimensional Fourier transform of the GPD, i.e. using the function $q(x, \mathbf{b})($ Fig $\mathbf{7})$. We pointed out that $\langle\mathbf{r}\rangle^{1 / 2} \rightarrow\langle\mathbf{y}\rangle^{1 / 2}$ for $x \rightarrow 1$, where $\mathbf{y}$ is the variable defining the radius in Ref. [37], and $\langle\mathbf{r}\rangle^{1 / 2} \rightarrow\langle\mathbf{b}\rangle^{1 / 2} \neq\langle\mathbf{y}\rangle^{1 / 2}$ for $x \rightarrow 0$. We unraveled the following behavior (Fig 8):

i) $\left\langle\mathbf{r}^{2}\right\rangle^{1 / 2}$ diverges for $x \rightarrow 0,1$ in 35 .

ii) $\left\langle\mathbf{r}^{2}\right\rangle^{1 / 2} \rightarrow 0$ for $x \rightarrow 1$ in [37]. 
iii) $\left\langle\mathbf{r}^{2}\right\rangle^{1 / 2}$ decreases from the value of $\approx 5 \mathrm{GeV}^{-1}(\approx 1 \mathrm{fm})$, at $x \rightarrow 0$, to $\approx 2.5 \mathrm{GeV}^{-1}$ $(\approx 0.5 \mathrm{fm})$, at $x \rightarrow 1$ in our model.

From the behavior of the rms radius with $x$, one can surmise what components in $b$ space the form factor is dominated by. While $\left\langle\mathbf{r}^{2}\right\rangle^{1 / 2}$ at $x>0.5$ is entirely given by $b \lesssim 2.5 \mathrm{GeV}^{-1}$ $(0.5 \mathrm{fm})$ for models characterized by a hard component (ii) and iii)), in case $i)$ saturation is not reached (Fig.10), that is the rms radius is large even at large $x$. On the other side, by studying the average value of $x, x_{\text {ave }}$, calculated using the distribution $H$ vs. $\Delta$ (Figs 11 and (12), one finds that the hard type functions are not dominated by large $x$ at large $\Delta$, rather all $x$ components seem to contribute (Fig 12).

In summary, although it is becoming clear that the physics governing the hadronic form factors, and more generally exclusive processes at large momentum transfer, is given by a non-trivial blend of soft and hard components, current descriptions using GPDs are not completely satisfactory.

The next question is how one would be able to test the transverse spatial structure of hadrons, given the fact that GPDs are quite elusive objects from the experimental point of view. A potentially powerful means to single out the size of hadronic configurations could be given by combining CT/nuclear filtering studies with GPDs as suggested in this paper. Based on the so far little explored concepts of IPPDFs and GPDs, we can now test the effect of nuclear filtering in an exclusive reaction by building a "filter" in the definition of the transparency, $T_{A}$. This procedure eliminates some of the model dependence implicit in many predictions in a somewhat similar way to what proposed e.g. in Ref. [43]. It however also provides a way of explicitly extracting the transverse radial dependence of the hadronhadron cross section in the nuclear medium. While an analysis extended to all available data will be carried out in a upcoming paper [24], we have presented here a few hypothetical scenarios obtained with our procedure.

In addition to the main results of this paper, interesting new developments will concern the extension of our calculations to the neutron and the polarized case, as well as the exploration of the skewedness, $\xi$, dependence. Most importantly, the use of nuclei and CT experiments might unravel an alternative method to measure GPDs by evaluating nuclear dependent contributions beyond the standard assumption of factorization into a nucleon times a nuclear part for the nuclear scattering amplitude. Such terms can be shown to be directly proportional to GPDs and a study examining their relative importance as $A$ dependent contributions is on its way [24]. Both the studies initiated in this paper and the suggested future studies could be tested ideally both at Jefferson Lab [42] including its 12 GeV upgrade, and at newly planned Electron Ion Colliders with high luminosity and energy 47.

In conclusion, studies like the one presented here using the new concept of GPDs, will both improve our knowledge of nuclear filtering phenomena and allow for a more detailed understanding of the transverse components involved at large momentum transfer. In particular, we hope to have provided a connection between $\mathbf{b}-$ and $\mathbf{k}-$ space that will help to systematically address both the role of Sudakov effects in the endpoints of the hadronic wave function [5, 16], and the role of power corrections in the large longitudinal momentum regions hinted in [37]. 


\section{Acknowledgments}

We thank Karo Oganessyan for comments on the manuscript. This work is supported by the U.S. Department of Energy grant no. DE-FG02-01ER41200.

\section{APPENDIX A}

The coefficients defining $H(x, \boldsymbol{\Delta})$ in Eq.(26) are given by:

$$
\begin{aligned}
& p 1=2.780 x^{0.2}(1-x)^{4.2}\left(1+0.5 x^{0.3}\right) \\
& p 2=\left\{\begin{array}{c}
0.5695-0.1896 x+0.6885 x^{2} \quad x \leq 0.4 \\
0.605 x>0.4
\end{array}\right. \\
& p 3=1.118 x^{-0.098}(1-x)^{2.24} \\
& p 4=\left\{\begin{array}{c}
0 x \leq 0.87 \\
-0.1076 x+0.1079 x>0.87
\end{array}\right.
\end{aligned}
$$

[1] S. J. Brodsky and A. H. Mueller, Phys. Lett. B 206, 685 (1988).

[2] S. J. Brodsky, arXiv:hep-ph/0311355, and references therein.

[3] P. Jain and J. P. Ralston, Pramana 57, 433 (2001) arXiv:hep-ph/0103131.

[4] J. P. Ralston and B. Pire, Phys. Rev. Lett. 65, 2343 (1990).

[5] B. Kundu, J. Samuelsson, P. Jain and J. P. Ralston, Phys. Rev. D 62, 113009 (2000); ibid AIP Conf. Proc. 549, 455 (2002) arXiv:hep-ph/0008194.

[6] A. S. Carroll et al., Phys. Rev. Lett. 61, 1698 (1988); N. Makins et al., Phys. Rev. Lett. 72, 1986 (1994); T. G. O’Neill et al., Phys. Lett. B 351, 87 (1995); K. Garrow et al., Phys. Rev. C 66, 044613 (2002).

[7] P. Jain, B. Pire and J. P. Ralston, Phys. Rept. 271, 67 (1996)

[8] L.L. Frankfurt, G.A. Miller, M.I. Strikman, Ann. Rev. Nucl. Part. Sci. 44, 501 (1994); ibid, Comm. Nucl. Part. Phys., 21 1, (1992).

[9] N.N. Nikolaev, Comm. Nucl. Part. Phys., 21, 41 (1992).

[10] P. Jain, B. Kundu and J. P. Ralston, Phys. Rev. D 65, 094027 (2002).

[11] B.Z. Kopeliovich et al., Phys. Rev. C65, 035201 (2002).

[12] A. B. Borissov [HERMES Collaboration], Nucl. Phys. A 711, 269 (2002).

[13] L. Y. Zhu et al. [Jefferson Lab Hall A Collaboration], Phys. Rev. Lett. 91, 022003 (2003).

[14] D. Dutta et al. [Jefferson Lab E940104 Collaboration], Phys. Rev. C 68, 021001 (2003).

[15] E.M. Aitala et al., Phys. Rev. Lett. 86, 4773 (2001).

[16] P. Hoyer, J. T. Lenaghan, K. Tuominen and C. Vogt, arXiv:hep-ph/0210124.

[17] M. Burkardt, Int. J. Mod. Phys. A 18, 173 (2003) arXiv:hep-ph/0207047.

[18] M. Diehl, Eur. Phys. J. C 25, 223 (2002) [Erratum-ibid. C 31, 277 (2003)]

[19] A. V. Belitsky, X. d. Ji and F. Yuan, arXiv:hep-ph/0307383.

[20] X. D. Ji, Phys. Rev. Lett. 78, 610 (1997)

[21] A. V. Radyushkin, Phys. Lett. B 380, 417 (1996).

[22] K. Goeke, M . V. Polyakov and M. Vanderhaeghen, Prog. Part. Nucl. Phys. 47, 401 (2001)

[23] M. Diehl, Phys. Rept. 388, 41 (2003). 
[24] S. Liuti and S. K. Taneja, in preparation.

[25] D. E. Soper, Phys. Rev. D 15, 1141 (1977).

[26] S. J. Brodsky, P. Hoyer, N. Marchal, S. Peigne and F. Sannino, Phys. Rev. D 65, 114025 (2002).

[27] J. P. Ralston and B. Pire, Phys. Rev. D 66, 111501 (2002).

[28] G. P. Lepage, S. J. Brodsky, T. Huang and P. B. Mackenzie, CLNS-82/522, Invited talk given at Banff Summer Inst. on Particle Physics, Banff, Alberta, Canada, Aug 16-28, 1981.

[29] F. Gross and S. Liuti, Phys. Rev. C 45, 1374 (1992).

[30] R. Jakob, P. J. Mulders and J. Rodrigues, Nucl. Phys. A 626, 937 (1997), and references therein.

[31] S. A. Kulagin, G. Piller and W. Weise, Phys. Rev. C 50, 1154 (1994).

[32] P. Kroll, Proceedings of the workshop on "Exclusive processes at high momentum transfer, Editors A. Radyushkin and P. Stoler, World Scientific (2002), pp.214-224; arXiv:hep-ph/0207118, and references therein.

[33] A. Gardestig, A. P. Szczepaniak and J. T. Londergan, Phys. Rev. D 68, 034005 (2003).

[34] M. Gluck and E. Reya, Nucl. Phys. B 130, 76 (1977).

[35] A. V. Radyushkin, Phys. Rev. D 58, 114008 (1998).

[36] P. Stoler, Phys. Rev. D 65, 053013 (2002).

[37] M. Burkardt, arXiv:hep-ph/0401159.

[38] A. Airapetian et al. [HERMES Collaboration], Phys. Rev. Lett. 87, 182001 (2001).

[39] S. Stepanyan et al. [CLAS Collaboration], Phys. Rev. Lett. 87, 182002 (2001)

[40] S. Nussinov, Phys. Rev. Lett. 34, 1286 (1975).

[41] G. Bertsch, S. J. Brodsky, A. S. Goldhaber and J. F. Gunion, Phys. Rev. Lett. 47, 297 (1981).

[42] Jefferson Lab experimental proposal 01 - 107, K. Garrow and R. Ent, spokepersons.

[43] P. Jain and J. P. Ralston, Phys. Rev. D49, 1104 (1993).

[44] T. G. O’Neill et al., Phys. Lett. B 351, 87 (1995)

[45] K. Garrow et al., Phys. Rev. C 66, 044613 (2002).

[46] M. Burkardt and G. A. Miller, arXiv:hep-ph/0312190.

[47] "Second Electron-Ion Collider Workshop, Jefferson Lab, March 15-17, 2004.

http://www.jlab.org/intralab/calendar/archive04/eic/ 
(a)

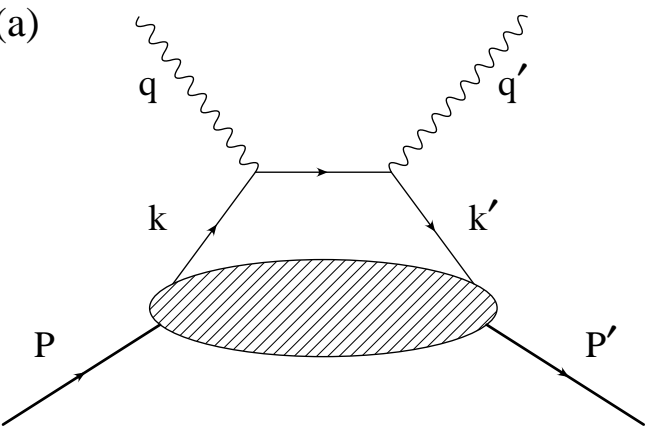

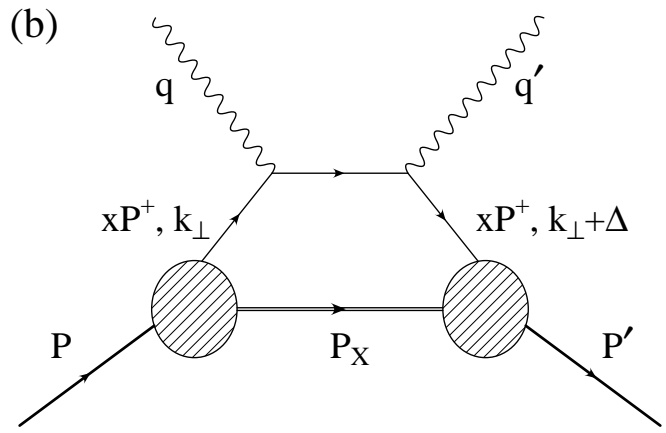

FIG. 1: (a) Amplitude for DVCS at leading order in $Q^{2}$; (b) The same amplitude in a two component model. 


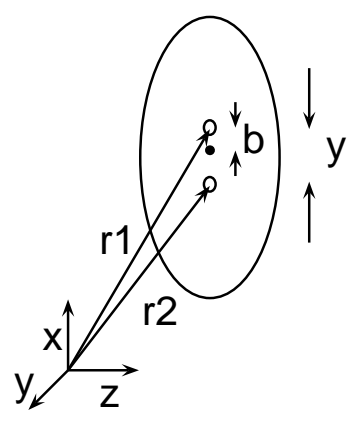

(a)

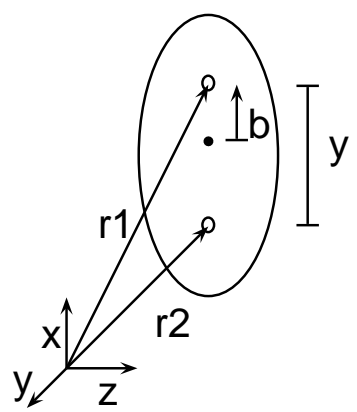

(b)

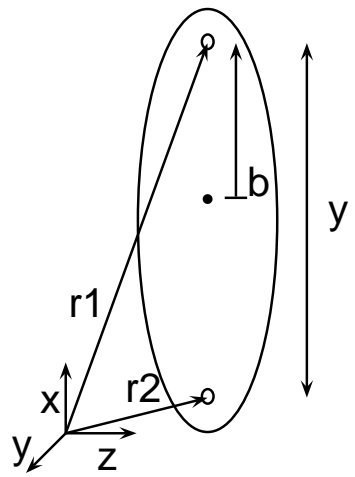

(c)

FIG. 2: The transverse distance between the active quark (upper blob) and the center of momentum of the spectator quarks (lower blob) - y; between the active quark and the center of momentum of the hadron (full circle) - b, and between the spectator quarks and the center of mass of the hadron $-\mathbf{b} x /(1-x) \equiv \mathbf{y}-\mathbf{b} . r_{1}$ and $r_{2}$ are the space vectors for the active quark and the center of momentum of the spectator quarks, respectively, taken in the system of coordinates $(x, y, z)$. For simplicity, $\mathbf{b}$ is drawn parallel to the $x$-axis. Assuming IPPDFs that yield values of $\left\langle\mathbf{b}^{2}\right\rangle$ decreasing as $x \rightarrow 1$, there are three possible behaviors for $\mathbf{y}$ and $\mathbf{r}$ (Eq.(10): (a) $\mathbf{b} \propto(1-x)^{\gamma}, \gamma>1$ $\Rightarrow \mathbf{y}, \mathbf{r}$ decrease as $x$ increases; (b) $\mathbf{b} \propto(1-x)^{\gamma}, \gamma=1 \Rightarrow \mathbf{y}, \mathbf{r}$ are constants independent of $x$; (c) $\mathbf{b} \propto(1-x)^{\gamma}, \gamma<1 \Rightarrow \mathbf{y}, \mathbf{r}$ increase as $x$ increases. 


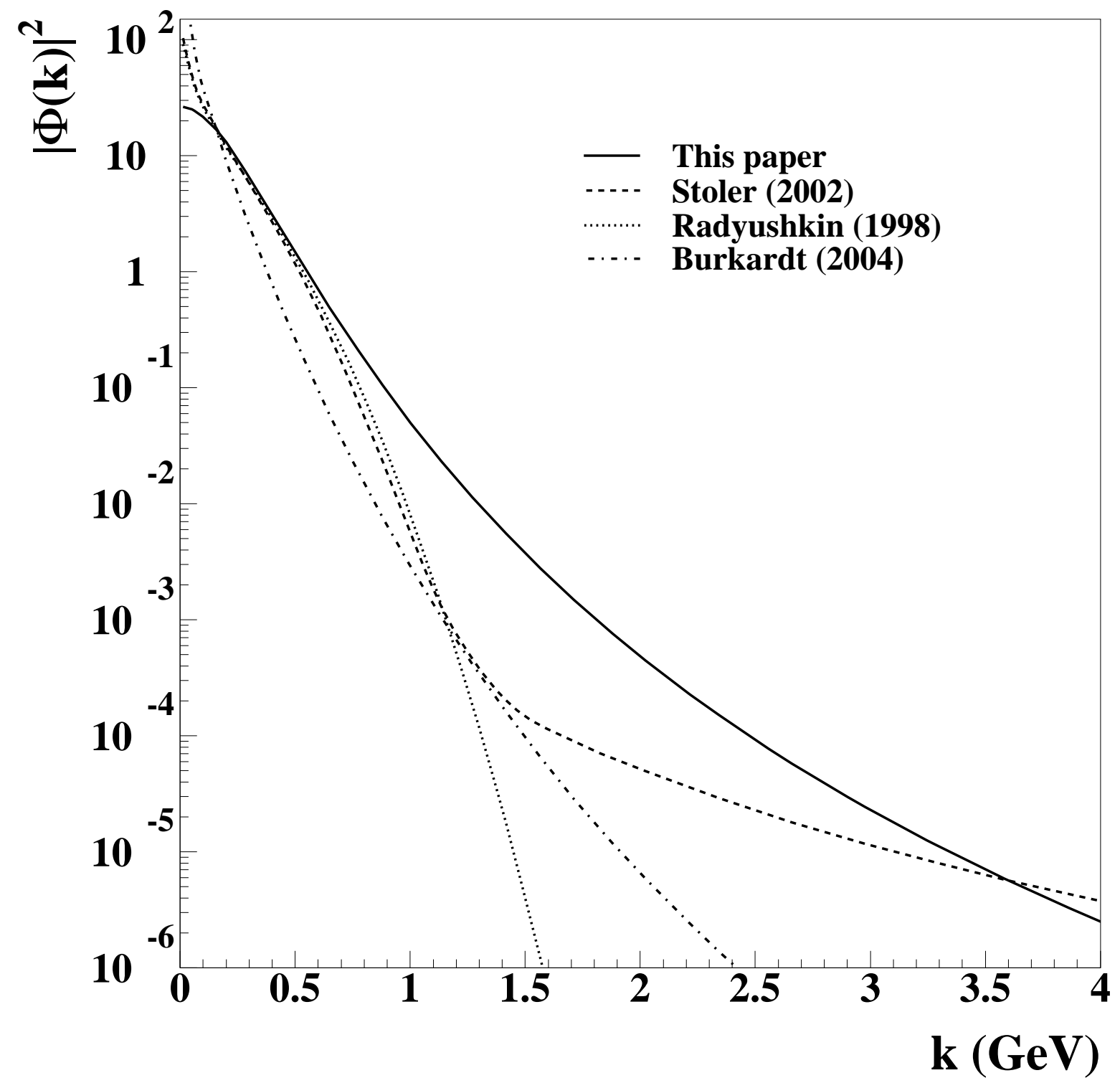

FIG. 3: The function $|\Phi(\mathbf{k})|^{2}$, defined in Eq.(24). Full line: model calculation discussed in this paper; dotted line: the soft wave function introduced in Ref. [35]; dashed line: model of Ref. 36., including a hard component in $\Phi$; dot-dashed line, the "semi-hard" distribution proposed in Ref. [37]. 


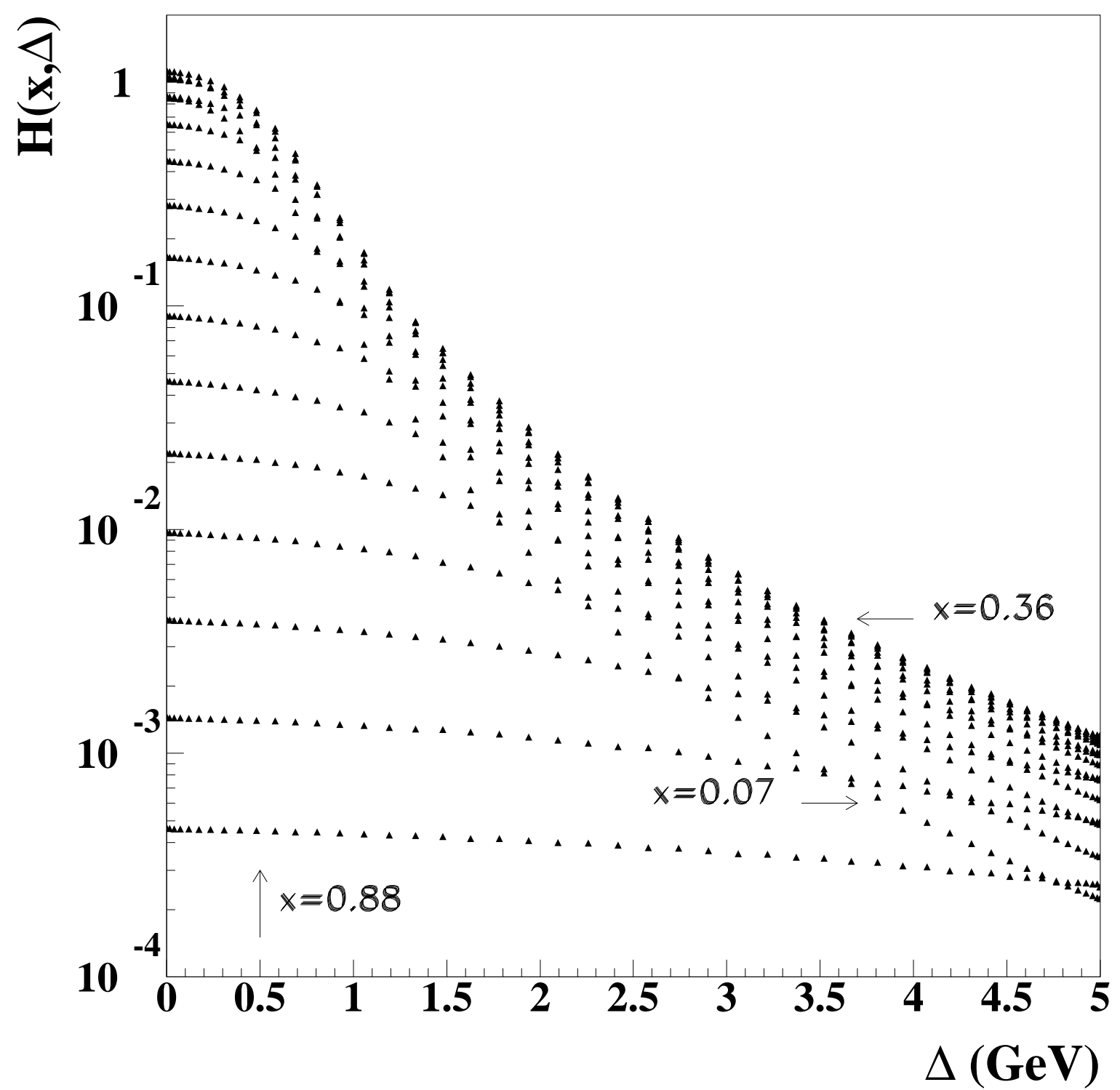

FIG. 4: The GPD, $H(x, \boldsymbol{\Delta})$, calculated according to Eqs.(22a) and (26), plotted vs. $\Delta$ for different values of $x$. Typical values, $x=0.07,0.36,0.88$, are shown by the arrows. 


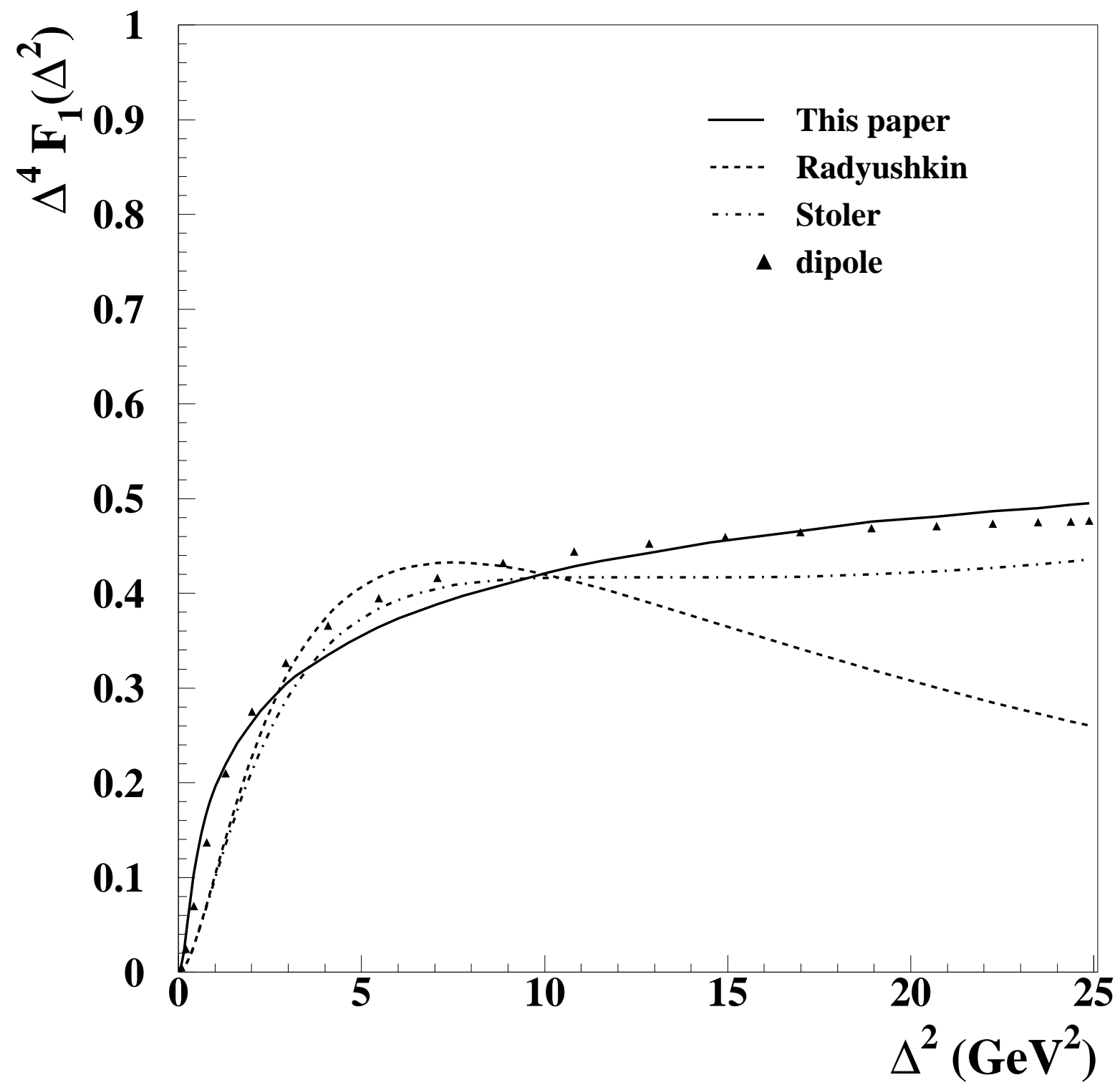

FIG. 5: The form factor, $F_{1}$ multiplied by $\Delta^{4}$, calculated in three different models: full line, this paper; dashed line, [35]; dot-dashed line, [36]. Results are compared with the dipole form factor (triangles). 


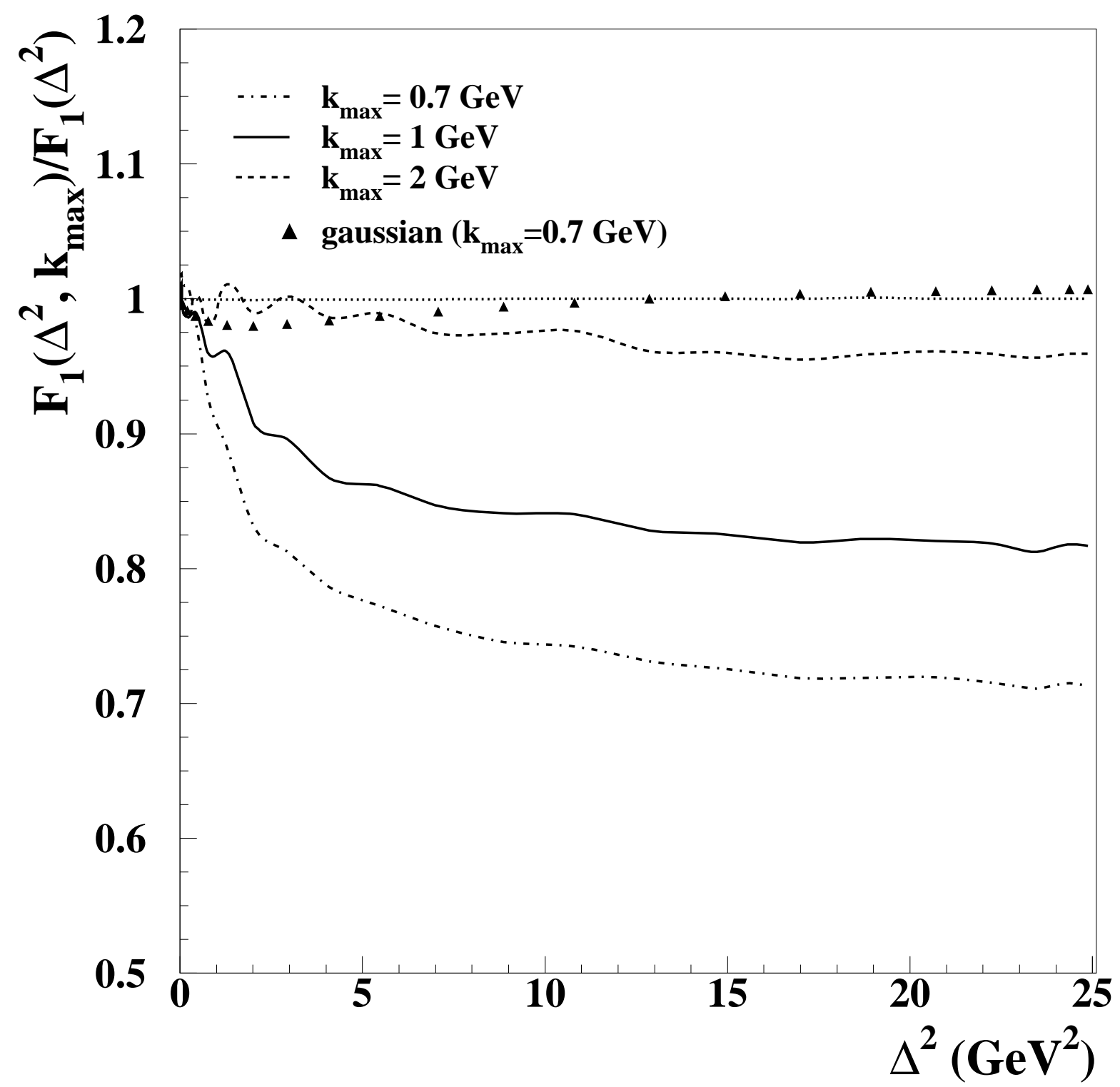

FIG. 6: Dominance of different $k$ components in $F_{1}\left(\Delta^{2}\right)$, showed using the ratio $F_{1}\left(\Delta^{2}, k_{\max }\right) / F_{1}\left(\Delta^{2}\right)$. The numerator was obtained by setting the upper limit of integration in Eq.(22c) to different values of $k \equiv k_{\max }$. The dot-dashed line is obtained for $k_{\max }=0.7 \mathrm{GeV}$; the full line corresponds to $k_{\max }=1 \mathrm{GeV}$, and the dashed line to $k_{\max }=2 \mathrm{GeV}$. The triangles, obtained by setting $k_{\max }=0.7$ in the model of Ref. 35], clearly show the saturation of the ratio at low values of $k$. 


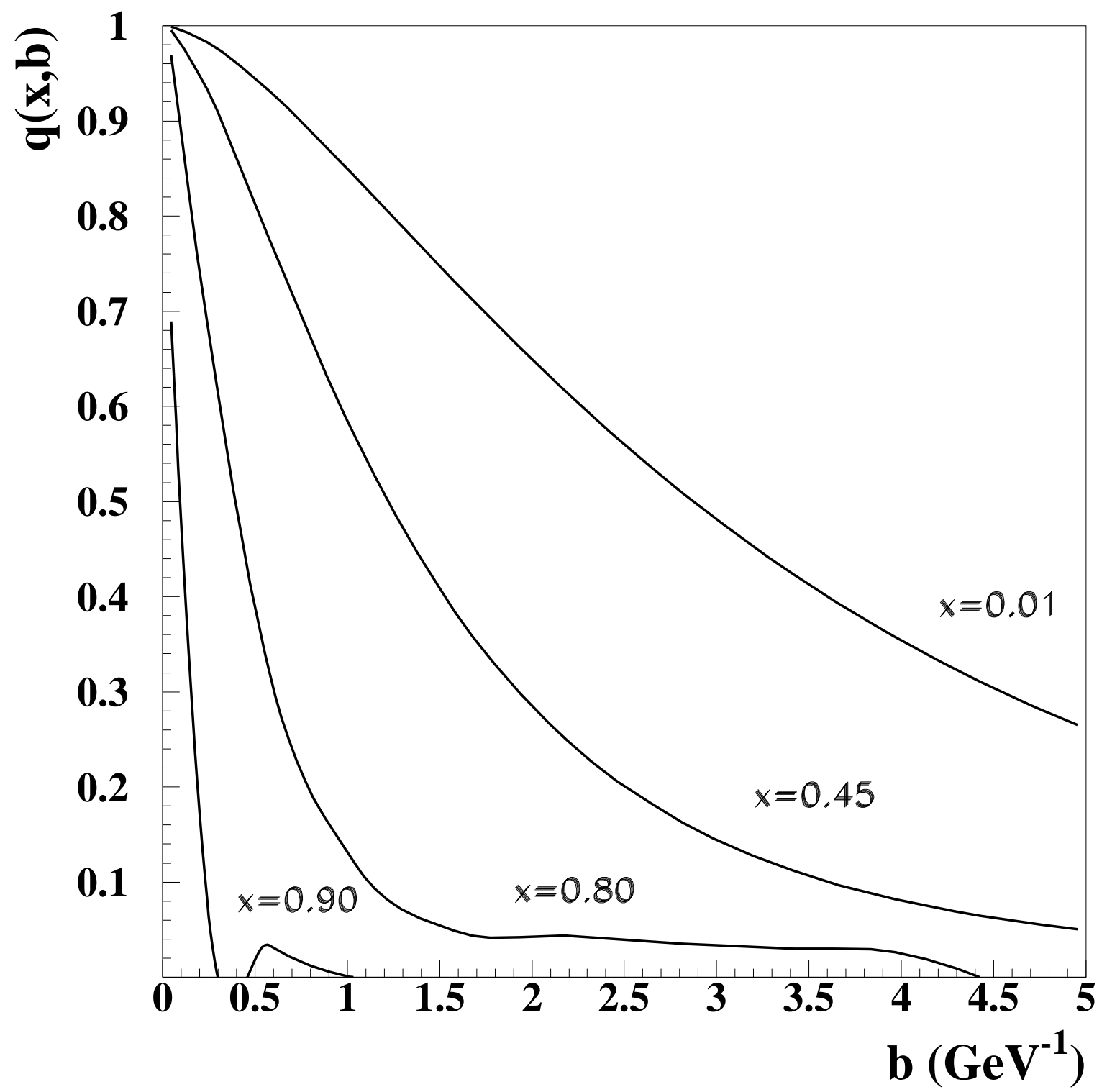

FIG. 7: IPPDF, $q(x, b)$ obtained by two-dimensional Fourier transforming Eq. 26), plotted vs. $b$. The curves shown in the figure correspond to different values of $x: 0.01,0.45,0.8,0.9$. 

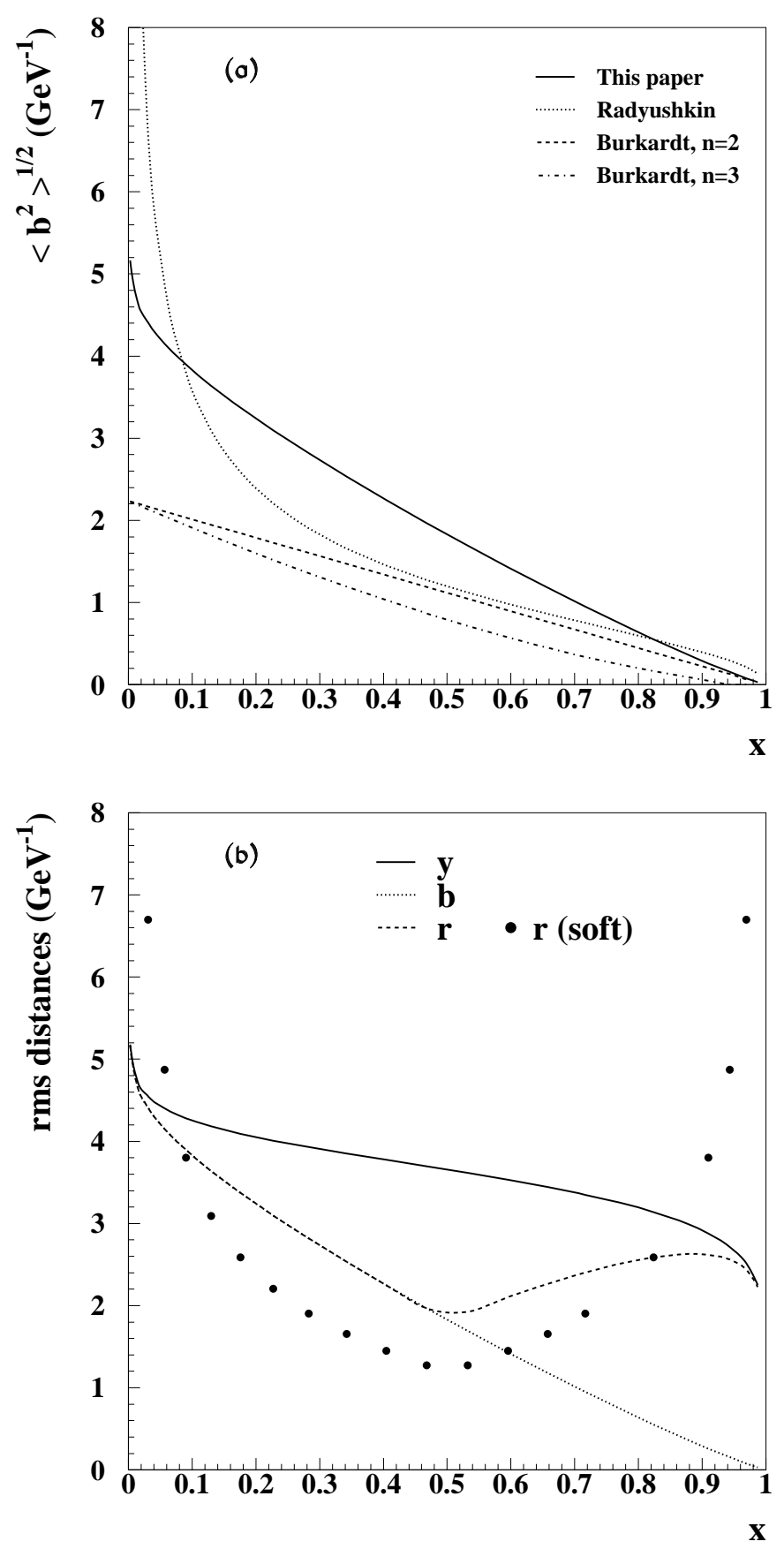

FIG. 8: Modeling the proton radius. (a) $\left\langle b^{2}\right\rangle^{1 / 2}$ in $\mathrm{GeV}^{-1}$, Eq.(15), plotted vs. $x$, calculated in different models: full line, this paper; dotted line 35]; the dashed and dot-dashed lines were calculated using the parametrization proposed in [37]. This parametrization describes the $\Delta^{2}$ behavior using a gaussian as in 35., however the argument which is $\propto(1-x) \Delta^{2}$ in 35] is suggested to be $(1-x)^{n} \Delta^{2}$, with $n \geq 2$ in [37]. The dashed line and the dot-dashed line were obtained by setting $n=2$ and $n=3$, respectively. (b) Transverse radial quantities: full line, $y \equiv\left\langle\mathbf{y}^{2}\right\rangle^{1 / 2}$, Eq.(9); dots, $b \equiv\left\langle\mathbf{b}^{2}\right\rangle^{1 / 2}$, Eq.(15), dashes, $r \equiv\left\langle\mathbf{r}^{2}\right\rangle^{1 / 2}$, Eq.(10). All quantities are calculated in the diquark model. For comparison $r$ calculated in the model of [35], based on a "soft" type wave function, is also shown. 


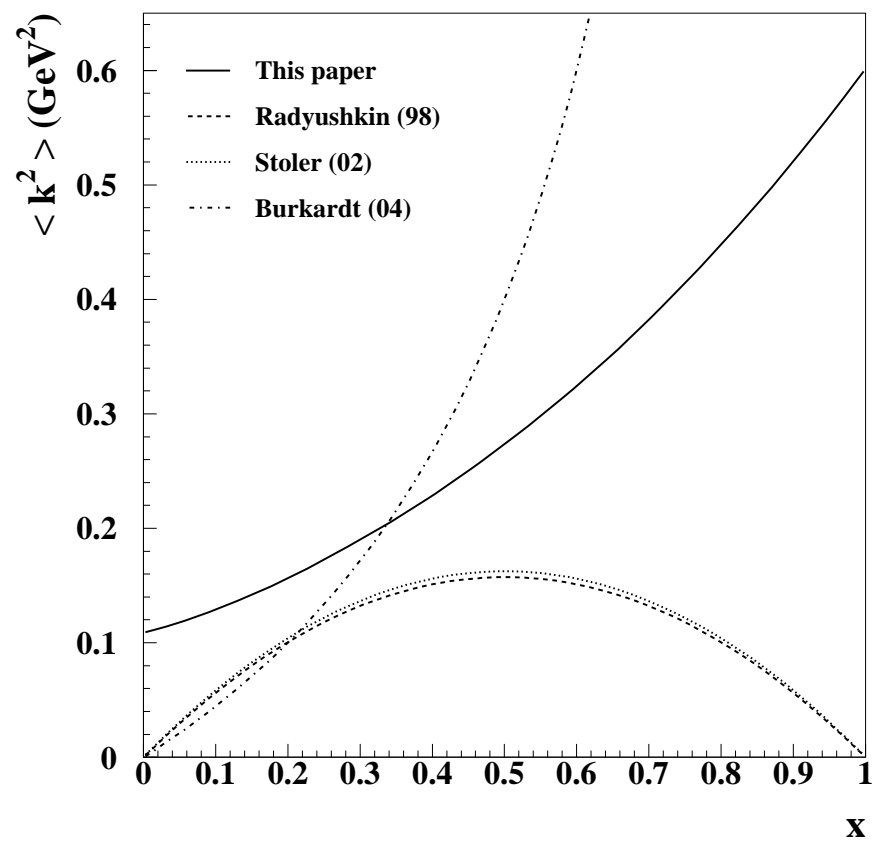

FIG. 9: Average intrinsic transverse momentum squared, Eq.16], plotted as a function of $x$ using different wave functions. Full line: model calculation discussed in this paper; dotted line: Ref. 35]; dashed line: Ref. [36]; dot-dashed line, the "semi-hard" distribution proposed in Ref. [37]. 

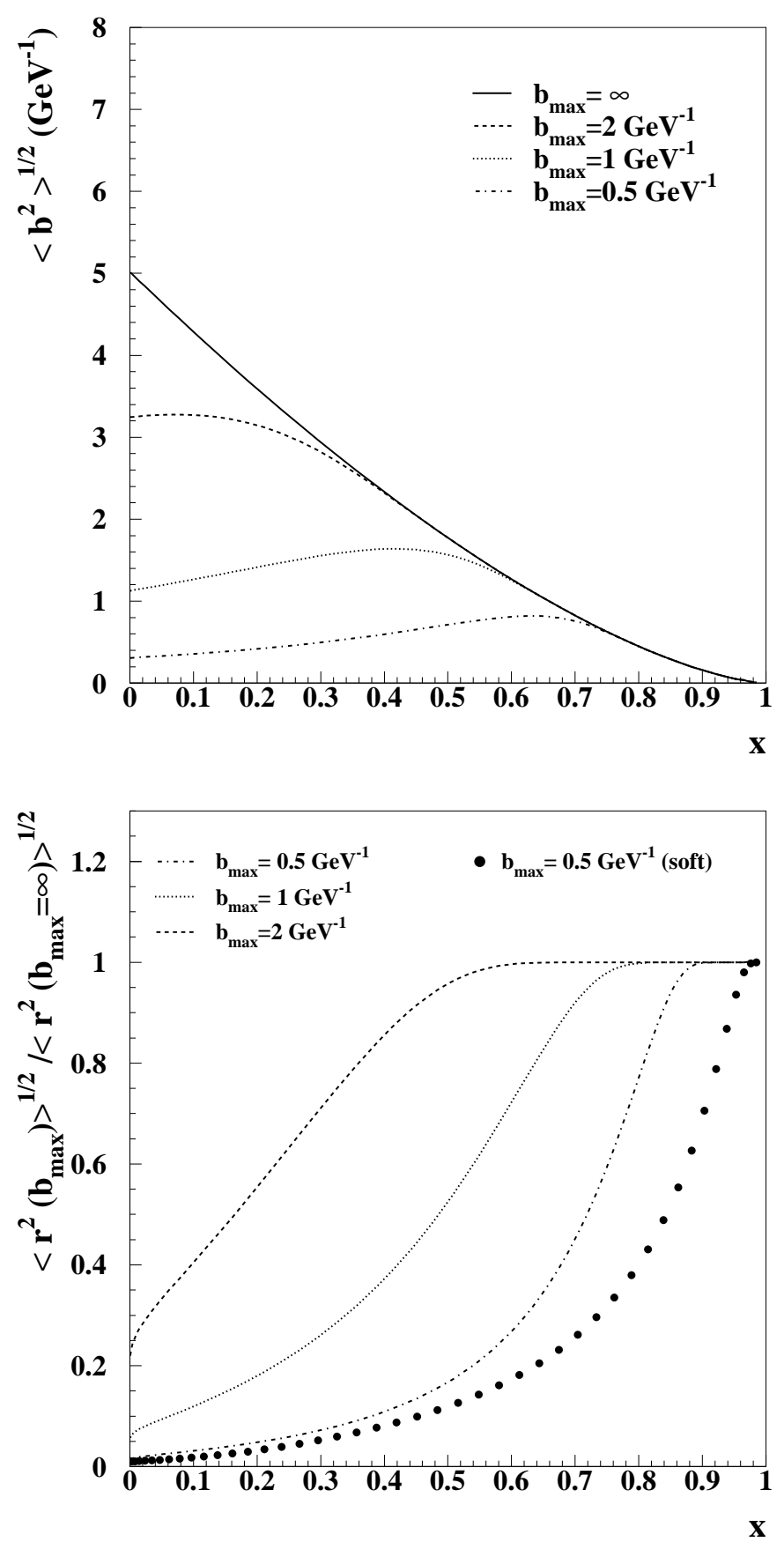

FIG. 10: Contribution of different transverse distances to the rms impact parameter $\left\langle b^{2}\right\rangle^{1 / 2}$, Eq. (5). Upper panel: Different contributions are calculated with the spectator/diquark model. They are evaluated setting the upper limit of integration in Eq.(5) to different values of $b \equiv b_{\max }$. The full line is obtained integrating over the whole range; the dashed line corresponds to $b_{\max }=2 \mathrm{GeV}^{-1}$; the dotted line to $b_{\max }=1 \mathrm{GeV}^{-1}$; the dot-dashed line to $b_{\max }=0.5 \mathrm{GeV}^{-1}$. Lower panel: ratio of the partial contributions displayed in the upper panel to the calculation using the full range of $b$. The continuous lines were obtained using the function in the upper panel. The big dots were obtained using the model of Ref. [35]. Notice that the ratio saturates much faster as a function of $x$ for the "hard" distribution adopted in this paper, than for the model of [35]. 


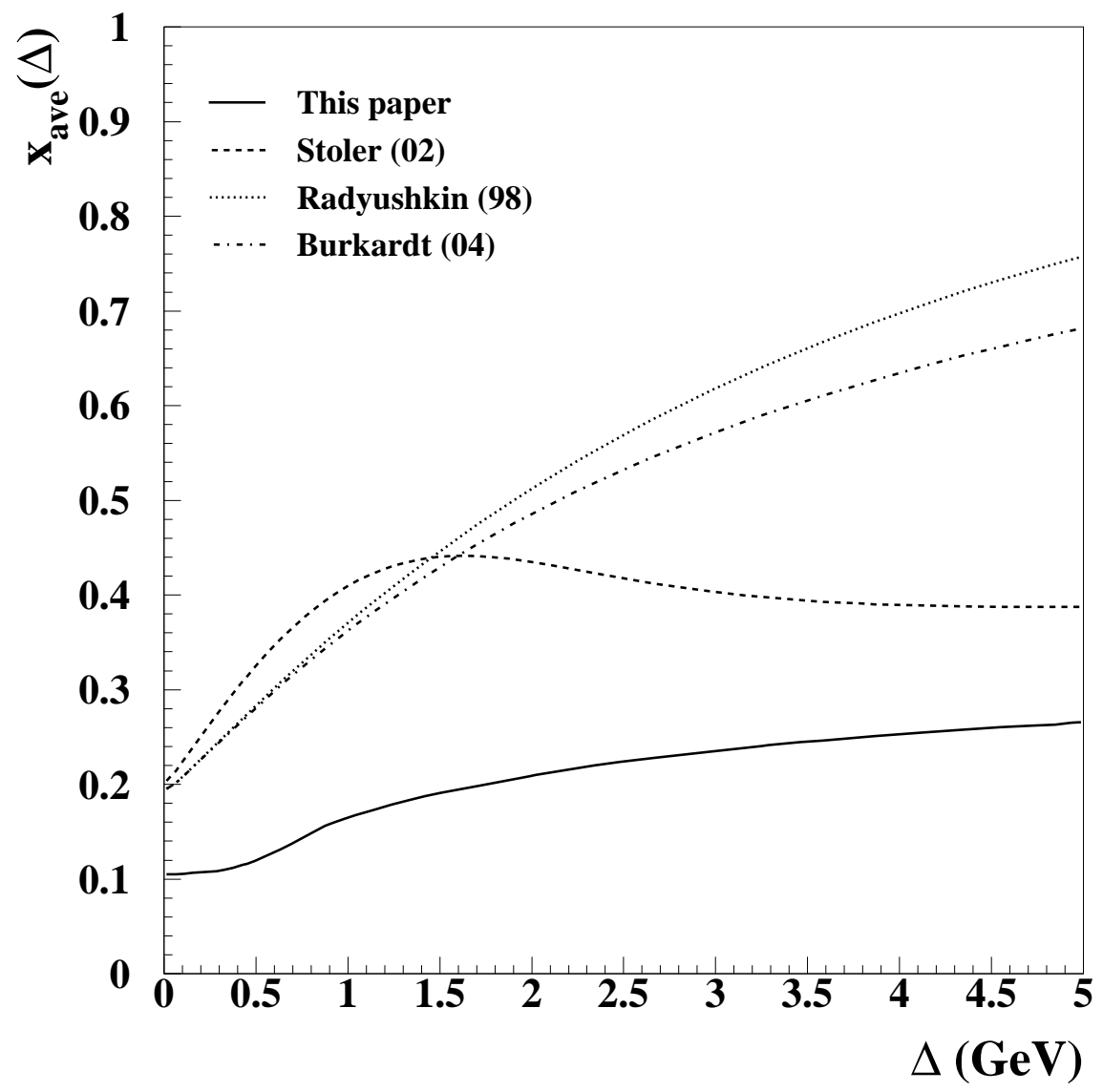

FIG. 11: Average value of $x, x_{\text {ave }}$, calculated using different wave functions in $H(x, \Delta)$ as a function of $\Delta$. The full line corresponds to the spectator model; the dashed line to the model of Ref. [36], the dotted line to Ref. 35], the dot-dashed line to Ref. [37], for $n=2$ (see also Fig [8). 


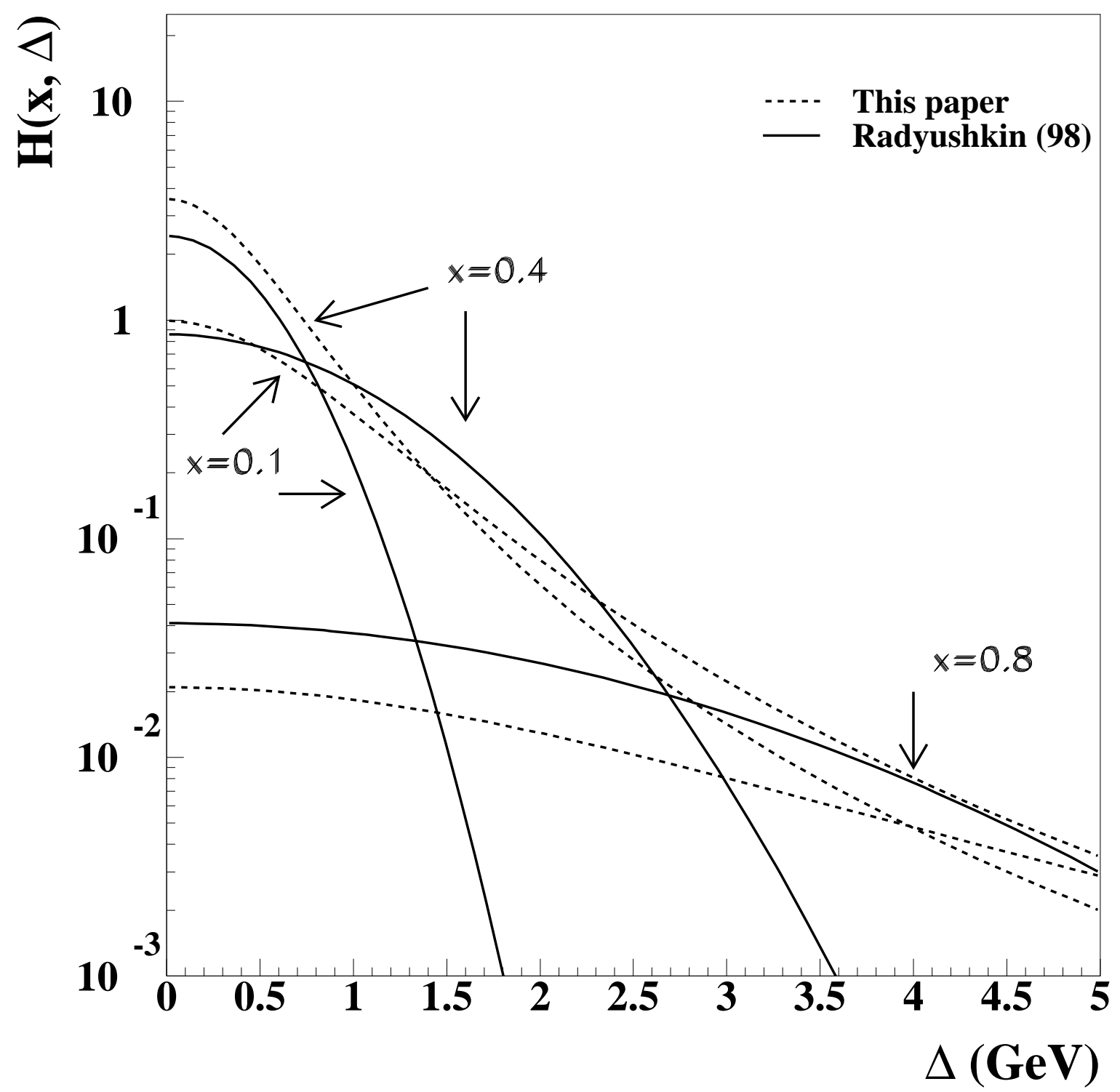

FIG. 12: Comparison of different $x$ components contributions to $H(x, \Delta)$ in the spectator model (full lines), and in the model of Ref. 35] (dashed lines). The latter, although being soft, shows the dominance of (small) large $x$ components at (small) large $\Delta$. In the spectator model, regardless of its hard $k$ components, also small values of $x$ contribute to large $\Delta$. The arrows in the figure indicate the three different values of $x$ for which the curves were calculated. 


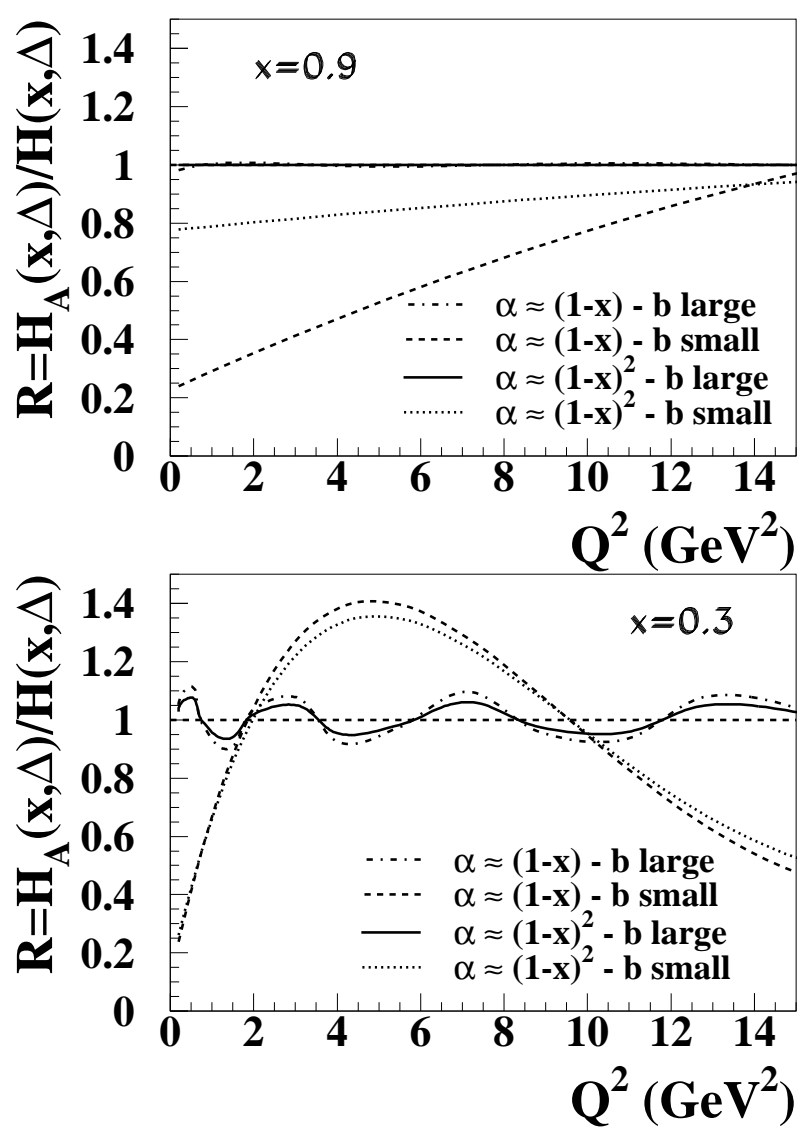

FIG. 13: Possible scenarios for the effect of nuclear filtering and the onset of CT based on a simplified model described in the text. The ratio $R$ of GPDs in a nucleus and in free space is plotted vs. $Q^{2} \equiv \Delta^{2}$, for two different values of $x: x=0.9$, upper panel and $x=0.3$, lower panel. For each panel we consider: (a) $\alpha \propto(1-x) / x$, as in Ref. 35]. (dot-dashed and dashed lines); (b) $\alpha \propto(1-x)^{2}$, as in Ref. 37] (full and dotted lines). Moreover, the full and dot-dashed line correspond to a large value of the filter's size, $b_{\max }$, the dashed and dotted lines to a small value of $b_{\max }$. The effect of the latter is to produce a suppression in the ratio, which is more pronounced at large $x$ for distributions with a large radius [35]. 